\title{
Exponentially light dark matter from coannihilation
}

\author{
Raffaele Tito D'Agnolo, ${ }^{a}$ Cristina Mondino, ${ }^{b}$ Joshua T. Ruderman ${ }^{b, c}$ \\ and Po-Jen Wang ${ }^{b}$ \\ ${ }^{a}$ SLAC National Accelerator Laboratory, \\ 2575 Sand Hill Road, Menlo Park, CA, 94025, U.S.A. \\ ${ }^{b}$ Center for Cosmology and Particle Physics, Department of Physics, \\ New York University, New York, NY 10003, U.S.A. \\ ${ }^{c}$ Theoretical Physics Department, CERN, \\ Geneva, Switzerland \\ E-mail: dagnolo@slac.stanford.edu, cm4001@nyu.edu, ruderman@nyu.edu, \\ pjw319@nyu. edu
}

ABSTRACT: Dark matter may be a thermal relic whose abundance is set by mutual annihilations among multiple species. Traditionally, this coannihilation scenario has been applied to weak scale dark matter that is highly degenerate with other states. We show that coannihilation among states with split masses points to dark matter that is exponentially lighter than the weak scale, down to the keV scale. We highlight the regime where dark matter does not participate in the annihilations that dilute its number density. In this "sterile coannihilation" limit, the dark matter relic density is independent of its couplings, implying a broad parameter space of thermal relic targets for future experiments. Light dark matter from coannihilation evades stringent bounds from the cosmic microwave background, but will be tested by future direct detection, fixed target, and long-lived particle experiments.

Keywords: Beyond Standard Model, Cosmology of Theories beyond the SM

ARXiv EPrint: 1803.02901 


\section{Contents}

1 Introduction 1

2 Relic density $\quad 4$

2.1 General discussion 4

$\begin{array}{lll}2.2 & \text { The relic density in a simple model } & 7\end{array}$

3 Annihilations to dark sector particles $\quad 10$

$\begin{array}{lll}3.1 & \text { Higgs portal } & 11\end{array}$

$\begin{array}{lll}3.2 & \text { Decoupled dark sector } & 15\end{array}$

4 Direct annihilations to standard model particles $\quad 17$

5 Conclusions 20

\section{Introduction}

Dark matter (DM) dominated the energy density of the Universe for much of its lifetime. Today it accounts for approximately a fifth of its energy budget. Its microscopic origin is unknown, but several theoretical possibilities have been identified. In this work we focus on thermal relics, whose present abundance is determined by the freeze-out of their interactions in the early Universe. This set of DM candidates has the attractive feature of having a relic density that is insensitive to initial conditions and is tied to potentially measurable couplings.

The standard paradigm for thermal relics is the so-called WIMP (Weakly Interacting Massive Particle) miracle [1-4]. In this scenario DM is kept in chemical equilibrium with the Standard Model (SM) thermal bath through its $2 \rightarrow 2$ annihilations. When the rate of its annihilations becomes slower than the expansion rate, DM acquires a non-zero chemical potential and its number density freezes-out, redshifting with the expansion of the Universe until today. The standard calculation points to DM with weak scale interactions

$$
\frac{\Omega_{\mathrm{WIMP}}}{\Omega_{\mathrm{DM}}} \approx \frac{(20 \mathrm{TeV})^{-2}}{\langle\sigma v\rangle} .
$$

However, increasingly stringent bounds on WIMPs from direct detection experiments [5-9], and the lack of obvious physics beyond the SM at the Large Hadron Collider, motivate exploration beyond the WIMP.

It has long been appreciated [10] that at least three "exceptions" exist to the standard WIMP computation: (1) mutual annihilation of multiple species (coannihilation), (2) annihilations into heavier states (forbidden channels) [11, 12], and (3) annihilations near a pole 

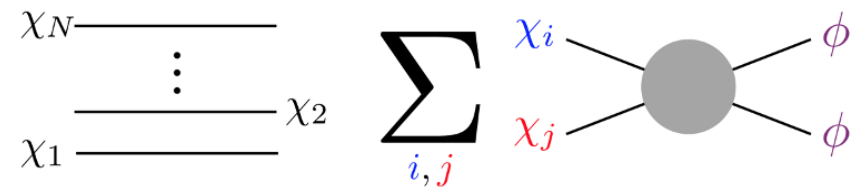

Figure 1. Illustration of the collection of $N$ states, $\chi_{i}$, required to realize coannihilation (left) and the processes that control DM freeze-out (right). DM annihilates into unstable states, $\phi$, from the thermal bath. The $N$ states share a quantum number and eventually decay to the lightest state, $\chi_{1}$, which constitutes the DM today. Freeze-out is determined by a summation over all possible annihilation modes among the collection of states.

in the cross section $[13,14]$. A fourth exception was recently identified where the DM abundance is set by inelastic scattering instead of annihilations (coscattering) [15-17]. These four exceptions retain most of the standard assumptions of the WIMP paradigm. DM starts in thermal equilibrium, has its number density diluted through $2 \rightarrow 2$ annihilations, and has the same temperature as SM photons at freeze-out.

The above exceptions correspond to small deformations of the assumptions underlying the WIMP miracle, and therefore correspond to close relatives of the WIMP within the broader theory space of DM candidates. However, the phenomenology of the above exceptions can differ dramatically from the WIMP. It was recently shown that forbidden channels and coscattering both naturally lead to DM that is exponentially lighter than the weak scale $[11,15]$. In this work we explore the best known among the above exceptions, coannihilation, and show that it also naturally leads to exponentially light DM. Other mechanisms leading to DM lighter than the weak scale are explored for example by refs. [18-32].

The dynamics of coannihilation are summarized in figure 1. Multiple states are assumed to share the DM stabilizing symmetry, and they can be ordered by increasing mass (left of figure 1), $m_{\chi_{1}}<m_{\chi_{2}}<\ldots<m_{\chi_{N}}$. In general, freeze-out is determined by a summation of all possible annihilation channels (right of figure 1). We postpone a technical discussion of the relic density till later, but for now note that ref. [10] assumed that DM has a weak scale mass, and found that coannihilation is only relevant for weak scale DM when the various states are highly degenerate, with mass splittings below $\sim 1-10 \%$. Weak scale coannihilation has been studied extensively in supersymmetry when the lightest neutralino is degenerate with another superpartner [33-52], and also within non-supersymmetric models [53-59]. Here we explore coannihilation among multiple non-degenerate particles, and show that coannihilation opens up parameter space where DM can be orders of magnitude lighter than a WIMP. This was first pointed out in [60] and later studied for specific models [61, 62].

There are several phases for (co)annihilation, summarized in figure 2, depending on which type of annihilations dominates at freeze-out. The left diagram shows the WIMP, where $\chi_{1} \chi_{1}$ annihilations dominate. The central diagram shows when coannihilation containing heavier particles in the set are important. The right diagram shows a special 


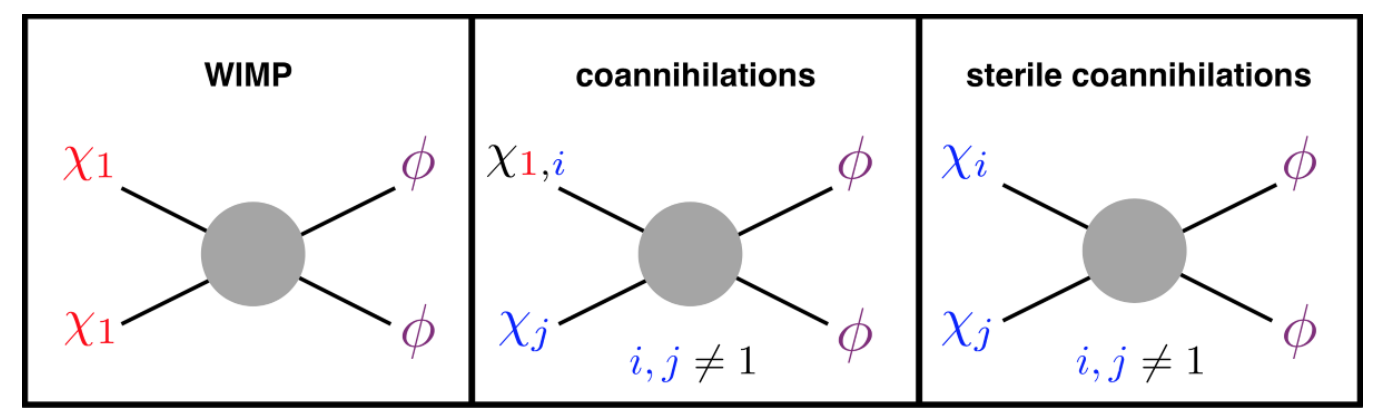

Figure 2. Schematic representation of three phases of coannihilation for a set of DM particles, $\chi_{i}$, sharing a conserved quantum number. At late times, the heavier states decay to the lightest one, $\chi_{1}$, which comprises the DM today. In the WIMP phase, $\chi_{1} \chi_{1}$ annihilations dominate. In the coannihilation phase, other annihilations dominate. In the sterile coannihilation phase, $\chi_{1}$ does not participate in the dominant annihilations, and therefore the relic abundance of DM is independent of its couplings.

phase of coannihilation, that we dub sterile coannihilation, where the dominant annihilation modes do not contain DM in the initial state. Sterile coannihilation manifests in supersymmetry when the bino coannihilates with a wino [48-50, 52], stop [38, 42], or gluino [46, 51], and in non-supersymmetric models [54, 56, 57, 59], but we are the first to apply it to light DM. Sterile coannihilation is our main focus in this paper, and has the special property that the DM relic density is independent of the couplings of DM. Experiments searching for light DM often focus on thermal relic targets [63], a subset of hidden-sector DM models where the DM was in thermal equilibrium with the SM in the early universe and freeze-out happens through DM annihilations into SM particles. In this case the DM coupling to the SM can be fixed by matching the observed abundance for each DM mass. These models are therefore highlighted for providing experiments with a bounded target of parameter space. Sterile coannihilation is still in the domain of thermal relics in kinetic equilibrium with the SM at freeze-out. However it relaxes the relation between dark matter couplings and abundance, pointing to a broader parameter space.

Coannihilation naturally evades stringent bounds from the Cosmic Microwave Background (CMB) [64] because, while all annihilations contribute at freeze-out, the heavier states will typically decay down to $\chi_{1}$ before recombination $[60,65]$. Therefore, the CMB is only sensitive to $\chi_{1} \chi_{1}$ annihilations, which may be suppressed if other annihilations dominate at freeze-out. This is illustrated in figure 3 , which shows the $\chi_{1} \chi_{1}$ annihilation cross section, for a particular model that we introduce below, as a function of the size of the mass mixing between DM, which begins with no interactions, and an active state that experiences rapid annihilations. At large mixing, DM is WIMP-like and excluded by the $\mathrm{CMB}$, whereas at small mixing coannihilation (or coscattering) sets the relic density and the CMB bound is evaded.

The remainder of this paper is organized as follows. In section 2.1, we review the formalism of ref. [10] for computing the relic density from coannihilation and we explain why coannihilation among multiple non-degenerate species naturally leads to DM that 


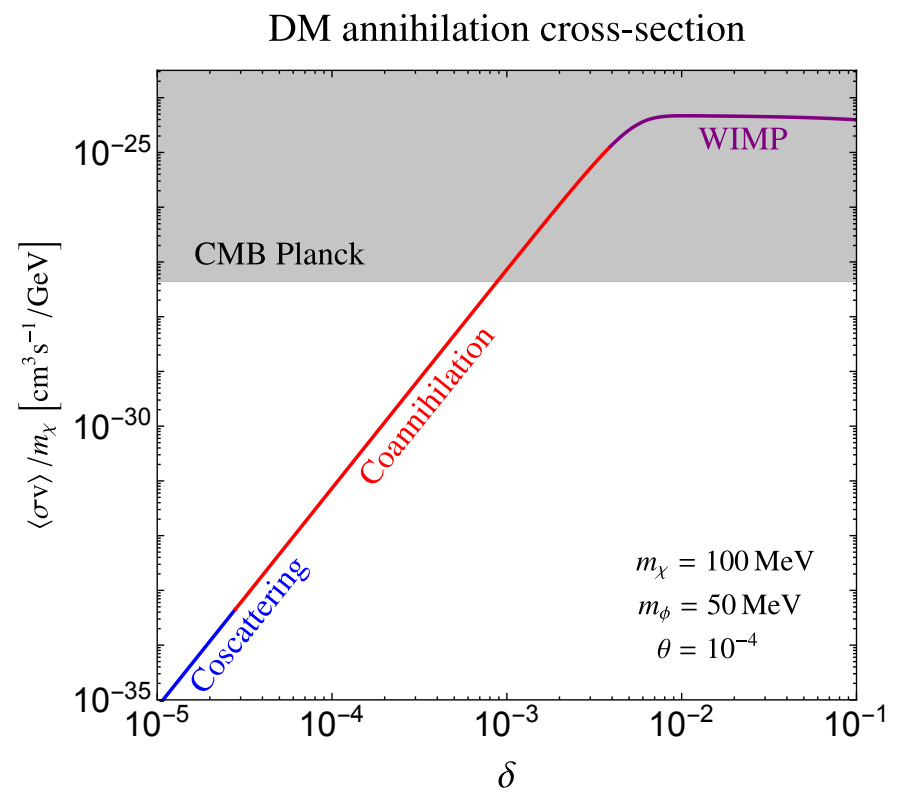

Figure 3. The DM self-annihilation cross section, $\chi \chi \rightarrow \phi \phi$, as a function of the mass mixing between mostly sterile DM, $\chi$, and an active state with rapid annihilations, $\psi$. The amount of mixing is measured using the parameter $\delta \equiv \delta m / m_{\chi}$, where $\delta m$ is the mass mixing between $\chi$ and $\psi$. The DM mass is fixed to $m_{\chi}=100 \mathrm{MeV}$. DM annihilates to a scalar, $\phi$, with mass $m_{\phi}=50 \mathrm{MeV}$ and mixing angle $\theta=10^{-4}$ with the SM Higgs. (See section 2.2 and 3.1 for a detailed description of the model and definitions of these parameters.) The color of the line delineates whether freeze-out is described by the WIMP (purple), coannihilation (red), or coscattering [15] (blue) phase. The gray region shows the Planck bound on DM annihilations [64], which excludes the WIMP phase for this DM mass.

is exponentially lighter than the weak scale. In section 2.2 , we introduce a toy model, with annihilations into dark states, that exhibits sterile coannihilation. In section 3 , we complete the toy model by coupling it to either the SM or a dark sector thermal bath, and we study the detailed phenomenology and experimental prospects. In section 4 , we consider an example model with direct annihilations to SM particles. section 5 contains our conclusions.

\section{Relic density}

\subsection{General discussion}

In this section, we begin by reviewing the formalism of ref. [10] for treating coannihilation. We then include a novel discussion of how non-degenerate coannihilation points to dark matter that is exponentially lighter than the weak scale.

We assume that DM is stabilized by a $\mathcal{Z}_{2}$ symmetry, or a larger symmetry with a $\mathcal{Z}_{2}$ subgroup. We consider a collection of $N$ particles, $\chi_{i}$, depicted in figure 1 , that include DM and have the same charge as DM under this stabilizing symmetry. We label them in such a way that $m_{\chi_{i}}<m_{\chi_{j}}$ for $i<j$. We take $\phi$ to stand for any other state that interacts with $\chi_{i}$ and is not charged under the $\mathcal{Z}_{2}$ symmetry. The relevant processes in the 
DM Boltzmann equations are: annihilations $\chi_{i} \chi_{j} \rightarrow \phi \phi$, inelastic scatterings $\chi_{i} \phi \rightarrow \chi_{j} \phi$, decays $\chi_{j} \rightarrow \chi_{i} \phi$, and the inverses of each of these processes. If the decays are sufficiently rapid, DM today is entirely composed of the lightest member of the set, $\chi_{1}$. We let $n_{i}$ refer to the number density of state $\chi_{i}$. The DM relic abundance is determined by the total number density, $n \equiv \sum_{i} n_{i}$, at freeze-out.

There are a priori $N$ separate Boltzmann equations, one for each of the $\chi_{i}$, but ref. [10] noticed an important simplification. As long as scatterings or decays are rapid enough to maintain chemical equilibrium among the different states, $\chi_{i} \leftrightarrow \chi_{j}$, the ratios of abundances track their equilibrium values, $n_{i} / n \approx n_{i}^{\text {eq }} / n^{\text {eq }}$, where $n_{i}^{\text {eq }}$ and $n^{\text {eq }}$ correspond to the equilibrium Boltzmann distributions. This means that $N-1$ Boltzmann equations can be removed, and a single Boltzmann equation governs the evolution of $n$. Coannihilation refers to this phase where scatterings and decays maintain chemical equilibrium until long after annihilations decouple, which is generically the case when $m_{\phi} \ll m_{\chi_{1}}$ and couplings are large enough. For massive $\phi$ or small enough couplings, exchange among the $\chi_{i}$ can decouple before annihilations, leading to the coscattering phase [15].

In the coannihilation limit, where $\chi_{i} \leftrightarrow \chi_{j}$ is rapid until after annihilations decouple, the evolution of $n$ in the early Universe is governed by the single Boltzmann equation

$$
\frac{d n}{d t}=-3 H n-\sum_{i, j=1}^{N}\left\langle\sigma_{i j} v\right\rangle\left(n_{i} n_{j}-n_{i}^{\mathrm{eq}} n_{j}^{\mathrm{eq}}\right)
$$

where $\left\langle\sigma_{i j} v\right\rangle$ are the thermally averaged annihilation cross sections (see figure 1). eq. (2.1) can be further simplified to

$$
\frac{d n}{d t}=-3 H n-\left\langle\sigma_{\mathrm{eff}} v\right\rangle\left(n^{2}-\left(n^{\mathrm{eq}}\right)^{2}\right)
$$

where we used $n_{i} / n \approx n_{i}^{\mathrm{eq}} / n^{\mathrm{eq}}$ and have defined an effective annihilation rate,

$$
\sigma_{\mathrm{eff}}=\sum_{i, j=1}^{N} \sigma_{i j} \frac{n_{i}^{\mathrm{eq}} n_{j}^{\mathrm{eq}}}{\left(n^{\mathrm{eq}}\right)^{2}} \approx \sum_{i, j=1}^{N} \sigma_{i j} \frac{g_{i} g_{j}}{g_{\mathrm{eff}}^{2}}\left(1+\Delta_{i}\right)^{3 / 2}\left(1+\Delta_{j}\right)^{3 / 2} e^{-x\left(\Delta_{i}+\Delta_{j}\right)},
$$

where $x=m_{\chi_{1}} / T, \Delta_{i} \equiv \frac{m_{\chi_{i}}-m_{\chi_{1}}}{m_{\chi_{1}}}$, and $g_{i}$ counts the number of internal degrees of freedom. The last step in eq. (2.3) holds when all $\chi_{i}$ 's are non-relativistic. As in [10], we find it convenient to define,

$$
g_{\mathrm{eff}} \equiv \sum_{i=1}^{N} g_{i}\left(1+\Delta_{i}\right)^{3 / 2} e^{-x \Delta_{i}}
$$

eq. (2.2) is the same as the Boltzmann equation for a single WIMP that leads to eq. (1.1), except the WIMP annihilation rate is replaced by the total effective annihilation rate. This implies that $\left\langle\sigma_{\text {eff }} v\right\rangle$ should be weak scale for coannihilation to reproduce the observed DM abundance. We see that heavy states decouple exponentially from the effective annihilation rate, because the $i j$ term is suppressed by the factor $e^{-x\left(\Delta_{i}+\Delta_{j}\right)}$. Ref. [10], and most of the following literature on coannihilation, have assumed that $\chi_{i}$ are weak scale particles and therefore that $\sigma_{i j}$ are weak scale. In order to prevent the exponential suppression from being 
too sizable, $\Delta_{i} \ll 1$ is required. This observation has led to the lore that coannihilation is only relevant among highly degenerate states. This is the most widely studied regime of coannihilation that found multiple applications in supersymmetric phenomenology. In a classic example the DM particles are a combination of nearly degenerate bino and wino or higgsino, and $\phi$ represents any light SM state, such as the electron [34, 40, 45, 47-50].

In this work we highlight a different regime, where non-degenerate coannihilation leads to light DM [60]. We assume that $\sigma_{11}$ is suppressed, so that $\sigma_{1 i}$ or $\sigma_{i j}$ dominates, with $i, j \neq 1$ (see the right panel of figure 2). We allow non-degeneracy: $\Delta_{i} \sim \mathcal{O}(1)$. In order for $\sigma_{\text {eff }}$ to be weak scale despite the exponential suppression, we require $\sigma_{i j}$ to be exponentially larger than the weak scale. This requires a mass scale for the dark states that is exponentially lighter than the weak scale, $m_{\chi_{i}} \ll \mathrm{TeV}$.

We will focus in particular on the limit of sterile coannihilation (right of figure 2). We imagine that the lightest particle in the DM family contributes negligibly to the total annihilation cross section:

$$
\sigma_{11}, \sigma_{1 i} \ll \sigma_{\text {eff }}
$$

Sterile coannihilation leads to the interesting simplification that the DM abundance depends on the DM mass, $m_{\chi_{1}}$ but is independent of the DM couplings and only depends on the annihilation rate of the heavier states and the mass splittings. At first it might seem surprising that annihilations that do not involve DM can deplete its abundance, but scattering and inverse decays allow $\chi_{1}$ to convert into heavier states, $\chi_{1} \rightarrow \chi_{i}$, such that the annihilations of heavy states effectively remove $\chi_{1}$ too.

We note that sterile coannihilation requires that DM be in chemical equilibrium with the heavier annihilating states, which places a lower limit on the strength of the interaction that is maintaining its chemical equilibrium. The relic density is parametrically independent of the size of the DM couplings as long as this lower bound is satisfied. If DM becomes weakly enough coupled that chemical equilibrium cannot be maintained, the relic density enters the coscattering phase of ref. [15]. We will see numerical examples below that highlight the transition from coannihilations to coscattering, and see also figure 4 of ref. [15].

To highlight the parametrics of sterile coannihilation, we make the further assumption that the heaviest state is the most strongly interacting and that the other annihilation channels can be neglected. Then the total cross section simplifies to

$$
\sigma_{\mathrm{eff}} \approx \frac{g_{N}^{2}}{g_{1}^{2}} \sigma_{N N}\left(1+\Delta_{N}\right)^{3} e^{-2 \Delta_{N} x}
$$

and the relic density acquires an exponential dependence on the mass splitting $\Delta_{N}$,

$$
\Omega_{\chi} h^{2} \propto \frac{1}{\left\langle\sigma_{\mathrm{eff}} v\right\rangle_{f}} \propto e^{2 \Delta_{N} x_{f}}
$$

where $x_{f}=m_{\chi_{1}} / T_{f}$ and $T_{f}$ is the temperature at which the heavy annihilations freezeout. The physical origin of this exponential is simple to understand. We have assumed that all the particles in the set are in equilibrium with each other (through scatterings or decays) until long after annihilations decouple. Therefore the chemical potential for $n$ is 
zero until the last annihilation process decouples from the thermal bath. In our example the last particle to decouple is also the heaviest. So freeze-out occurs earlier than it would if only the lightest state was present with the same interactions, $T_{f}=m_{N} / x_{f}>m_{1} / x_{f}$, implying an exponentially enhanced relic density for DM. The exponential in eq. (2.7) can be very large for $\mathcal{O}(1)$ mass splittings, allowing for dramatic departures from the standard WIMP lore.

If we fix the relic density to its observed value, in the sudden freeze-out approximation, $x_{f}$ is exactly the same as that of a WIMP and depends only on the DM mass. This is because for all thermal relics, the observed DM energy density today is,

$$
\rho_{\mathrm{DM}}=m_{\mathrm{DM}} n_{\mathrm{DM}}\left(x_{f}\right) \frac{s_{0}}{s_{f}},
$$

where $s_{0}$ and $s_{f}$ are the entropy densities today and at freeze-out, respectively. eq. (2.8) does not depend on the dynamics of freeze-out and is therefore universal for thermal relics, and allows $x_{f}$ to be solved as a function of $m_{\mathrm{DM}}$. Typical values of $x_{f}$ for thermal relics with weak scale masses are around 25 going down to about 10 at $10 \mathrm{keV}$, so $e^{-\Delta_{i} x_{f}}$ can be a large exponential suppression in sterile coannihilation.

We can also study $x_{f}$ without fixing the relic density, using the sudden freeze-out approximation, to see how $x_{f}$ depends on parameters such as the cross section and masses. In coannihilation freeze-out occurs when $n\left\langle\sigma_{\text {eff }} v\right\rangle \approx H$. If $\Delta_{i}=\left(m_{i}-m_{1}\right) / m_{1} \sim \mathcal{O}(1)$, when DM is non-relativistic the total number density is dominated by the lightest species ( $n \approx n_{1}$ ), since the heavier ones are exponentially suppressed. We can use the sudden freeze-out approximation to solve for $x_{f}$ in the usual way, with the only difference coming from the exponentials in $\sigma_{\text {eff }}$. In the previous example, where the $N$-th state dominates, we have (for $s$-wave annihilations)

$$
n_{1}\left\langle\sigma_{\mathrm{eff}} v\right\rangle \approx \frac{g_{N}^{2} m_{1}^{3}\left(1+\Delta_{N}\right)^{3}}{g_{1}(2 \pi x)^{3 / 2}} \sigma_{N N} e^{-\left(1+2 \Delta_{N}\right) x} .
$$

So $n_{1}\left\langle\sigma_{\text {eff }} v\right\rangle_{f} \approx H_{f}$ gives the right relic density at

$$
x_{f} \approx \frac{1}{1+2 \Delta_{N}}\left(21+\log \left[\frac{g_{N}^{2}\left(1+\Delta_{N}\right)^{3} m_{1} \sigma_{N N}}{g_{1} g_{*}^{1 / 2} \mathrm{GeV} \times \mathrm{pb}}\right]+\frac{1}{2} \log x_{f}\right),
$$

where $M_{\mathrm{Pl}}$ is the Planck mass and $g_{*}$ is the total number of relativistic degrees of freedom at freeze-out. As for the WIMP case, there is logarithmic dependence on the cross section and absolute mass scale, but unlike the case of the WIMP, there is also non-logarithmic dependence on the mass splitting through the factor $\left(1+2 \Delta_{N}\right)^{-1}$.

The numerical results that follow use MicrOMEGASv4 [66] to compute the relic density, unless otherwise specified. We have cross-checked MicrOMEGASv4 with our own numerical solutions of the Boltzmann equation and find good agreement.

\subsection{The relic density in a simple model}

To make some of these ideas more concrete we introduce a simple model where sterile coannihilation is realized. This model will serve as the main building block for our discussion 

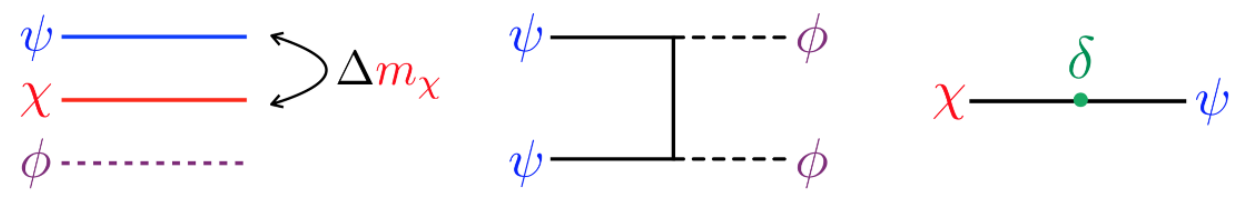

Figure 4. Schematic representation of the model described in section 2.2. From left to right: the mass spectrum, main annihilation channel, and strength of the mixing between DM, $\chi$, and its heavier coannihilating partner, $\psi$.
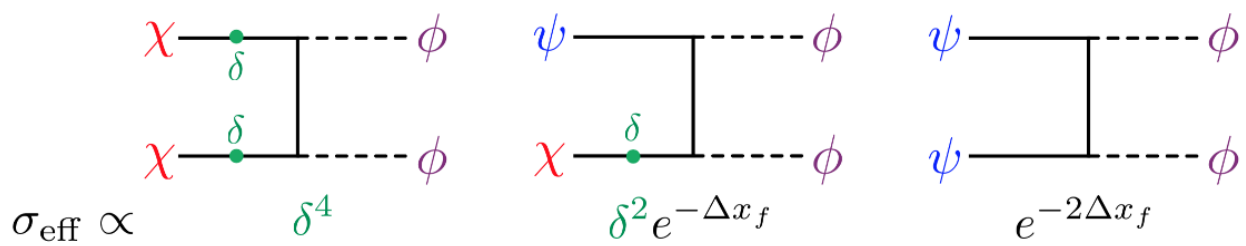

Figure 5. Annihilation channels for the model presented in section 2.2. The DM particle can approximately be identified with $\chi$. In the regime: $\delta^{2} \ll e^{-\Delta x_{f}}, \psi \psi$ annihilations dominate and we realize sterile coannihilation. In the opposite limit, $\delta^{2} \gg e^{-\Delta x_{f}}, \chi \chi$ annihilations dominate and the model becomes WIMP-like.

of coannihilation in dark sectors weakly coupled to (or decoupled from) the SM. It is a toy model (in this section) in the sense that we do not specify a coupling to radiation, which can take different forms and will determine the phenomenology of the theory, as we discuss in the next section.

We consider a Majorana fermion, $\psi$, which will act as the heavy particle that dominates the annihilation rate. We take $\psi$ to have rapid annihilations into a scalar particle, $\phi$, $\psi \psi \rightarrow \phi \phi$. Then we add DM in the form of a Majorana fermion, $\chi$, which begins as an inert particle and inherits interactions through a small mass-mixing $\delta m$ with $\psi$. In the small mixing limit, annihilations with $\chi$ in the initial state are suppressed, so that $\psi \psi$ annihilations naturally dominate. This setup is achieved by the following potential,

$$
V=\frac{m_{\phi}^{2}}{2} \phi^{2}+\frac{m_{\psi}}{2} \psi^{2}+\frac{m_{\chi}}{2} \chi^{2}+\delta m \chi \psi+\frac{y}{2} \phi \psi^{2}+\text { h.c. . }
$$

There are two physical phases in $V$ that we choose to parametrize by allowing $\delta m$ and $y$ to be complex. Notice that for generic phases of these parameters, the relevant annihilation processes are $s$-wave. We take $|\delta| \equiv\left|\delta m / m_{\chi}\right| \ll 1$ so $\chi$ is mostly sterile and $m_{\psi} \gtrsim m_{\chi}>$ $m_{\phi}$. This choice of parameters realizes in a simple way our sterile coannihilation scenario with $\chi_{1} \approx \chi$ and $\chi_{2} \approx \psi$. This is shown schematically in figure 4 , where, going from left to right, we sketch the mass spectrum, main annihilation channel, and strength of the mixing between DM and its coannihilating partner.

We assume that $\phi$ has a small coupling with the SM or with an additional light dark state, sufficient to keep the DM in kinetic and chemical equilibrium with radiation at 

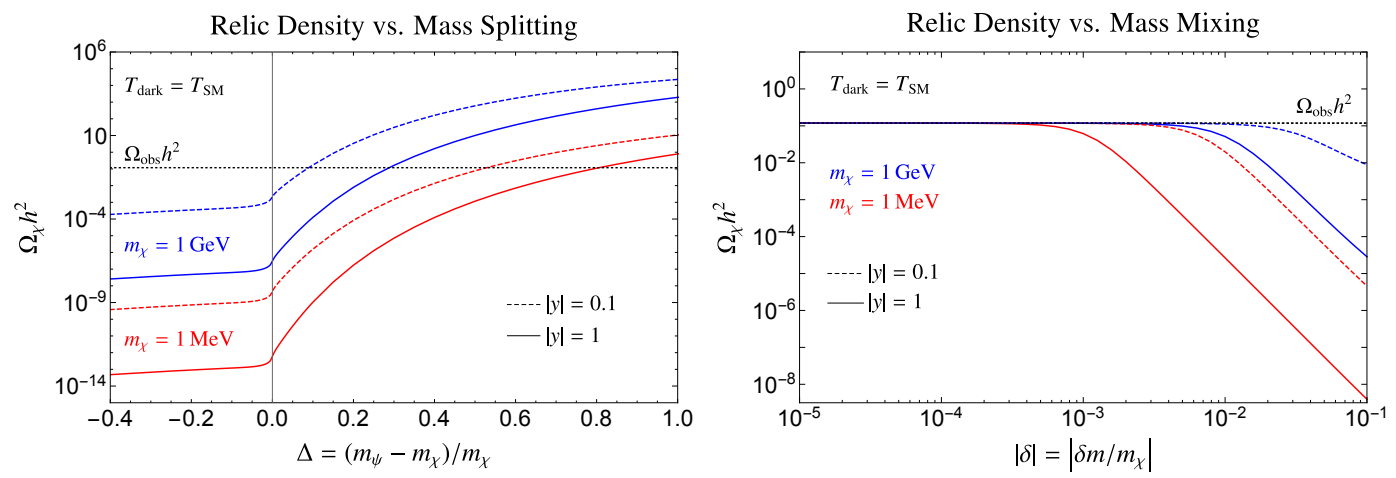

Figure 6. Left: dependence of the relic density on the mass splitting $\Delta$ between DM, $\chi$, and its rapidly annihilating partner, $\psi$. For $\Delta>0$ the relic density grows exponentially with the mass splitting, such that it crosses the observed value, $\Omega_{\mathrm{obs}} h^{2} \approx 0.12$, for a particular value of the splitting. Right: dependence of the relic density on the mass mixing, $\delta$, between DM and its partner, $\psi$. At large $\delta$ we exit the sterile coannihilation regime discussed in the text and lose the exponential dependence of the relic density on the mass splitting $\Delta$. Both figures were made for generic values of the phases: $y=|y| e^{i \pi / 4}$ and $\delta=|\delta| e^{i \pi / 4}$.

freeze-out, but small enough that annihilations of $\chi$ and $\psi$ to $\phi \phi$ dominate over direct annihilations to lighter states.

In figure 5, we show the three possible types of annihilations: $\chi \chi, \chi \psi$, and $\psi \psi$. When computing the effective annihilation rate, annihilations are suppressed by a factor of $\delta^{2}$ for each $\chi$ in the initial state, and by $e^{-x \Delta}$ for each $\psi$ in the initial state, where $\Delta \equiv$ $\left(m_{\psi}-m_{\chi}\right) / m_{\chi}$. Therefore, sterile coannihilation ( $\psi \psi$ dominating) is realized in the limit $\delta^{2} \ll e^{-\Delta x_{f}}$. In the opposite limit $\delta^{2} \gg e^{-\Delta x_{f}}$, the model becomes WIMP-like, with $\chi \chi$ annihilations dominating.

In figure 6 we illustrate the main qualitative point made in the previous section. The relic density grows exponentially with $\Delta \equiv\left(m_{\psi}-m_{\chi}\right) / m_{\chi}$. In the sterile coannihilation limit,

$$
\begin{aligned}
\left\langle\sigma_{\mathrm{eff}} v\right\rangle & \approx\left\langle\sigma_{\psi \psi} v(1+\Delta)^{3} e^{-2 \Delta x}\right\rangle \\
& =\frac{y_{r}^{2} y_{i}^{2}(1+\Delta)^{4}}{2 \pi m_{\chi}^{2}} \frac{\sqrt{(1+\Delta)^{2}-r^{2}}}{\left[2(1+\Delta)^{2}-r^{2}\right]^{2}} e^{-2 \Delta x}+\mathcal{O}\left(v^{2}\right)+\mathcal{O}\left(\delta^{2}\right),
\end{aligned}
$$

where $y_{r} \equiv \operatorname{Re}[y], y_{i} \equiv \operatorname{Im}[y]$, and $r \equiv m_{\phi} / m_{\chi}$. If the mass scale of the dark sector decreases or the coupling $y$ increases, the total cross section becomes larger, requiring a larger $\Delta$ to get the right relic density. This is shown in the left panel of figure 7 .

In the same figure we show that $x_{f}$ does not differ considerably from a WIMP and, as usual, decreases logarithmically with the DM mass. The last parameter of interest is the mixing $\delta$ between the DM, $\chi$, and the active state $\psi$. If it is sufficiently small, the relic density does not depend on it, as suggested by eq. (2.12). Only when we exit the sterile coannihilation regime does $\delta$ start to impact $\sigma_{\text {eff }}$. This is depicted in the right panel of figure 6 .

In figure 7 , when $m_{\chi}$ is below $1 \mathrm{MeV}$, we imagine that the dark sector is completely decoupled from the SM. (As we discuss below, limits from $N_{\text {eff }}$ exclude DM masses lighter 

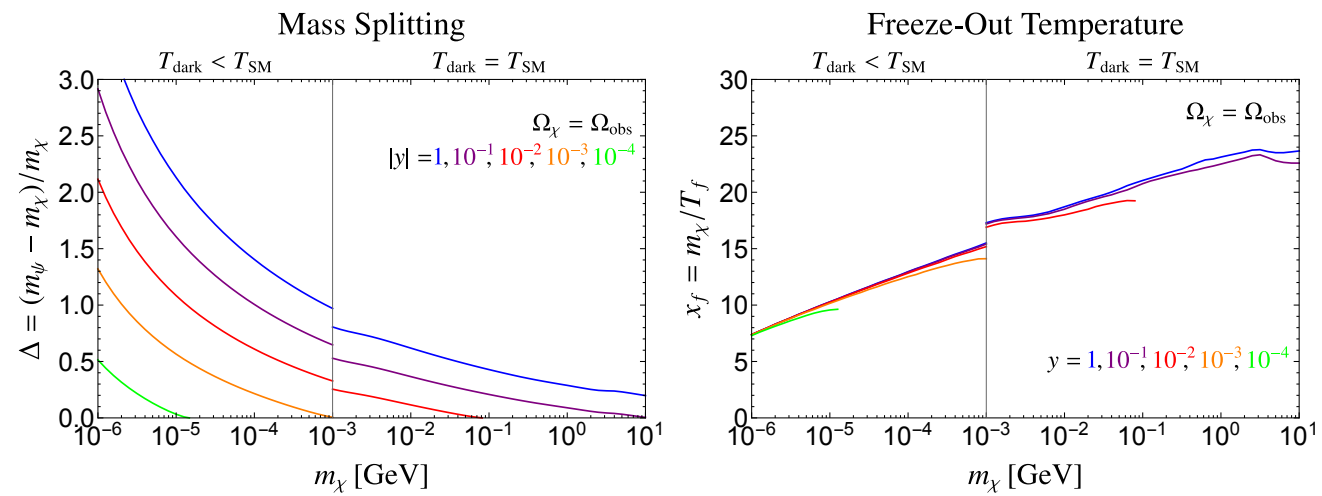

Figure 7. Left: value of the mass splitting, $\Delta$, that gives the observed relic density. We plot $\Delta$ as a function of $m_{\chi}$ and the coupling, $y$, controlling the size of the annihilation cross section. Light dark sectors require generic, $\mathcal{O}(1)$, mass splittings. Right: $x_{f}$ as a function of $m_{\chi}$ and the coupling $y . x_{f}$ in coannihilating dark sectors is the same as for a WIMP, after fixing the DM relic density to the observed value, and exhibits the same logarithmic dependence on the DM mass. Both figures were made for generic values of the phases: $y=|y| e^{i \pi / 4}$ and $\delta=|\delta| e^{i \pi / 4}$. For $m_{\chi}<\mathrm{MeV}$ we show the results for a decoupled dark sector in equilibrium with its own dark radiation (see section 3.2).

than the $\mathrm{MeV}$ scale when DM is in kinetic equilibrium with the SM.) In this case we add a light fermion species, with a sub-eV mass and coupling to $\phi$, that acts as dark radiation and insures the scaling $T_{\text {dark }} \sim a^{-1}$. In general, the SM and dark sectors evolve with separate temperatures, when they are kinetically decoupled, that are determined by the separate conservation of entropy in each sector [67]

$$
\frac{T_{\text {dark }}}{T_{\mathrm{SM}}}=\xi_{R}\left(\frac{g_{* S}^{\mathrm{SM}}\left(T_{\mathrm{SM}}\right)}{g_{* S}^{\mathrm{SM}}\left(T_{\mathrm{SM}}^{R}\right)} \frac{g_{* S}^{\mathrm{dark}}\left(T_{\mathrm{dark}}^{R}\right)}{g_{* S}^{\mathrm{dark}}\left(T_{\mathrm{dark}}\right)}\right)^{1 / 3},
$$

where $T_{\text {dark } S M}^{R}$ are the initial dark and SM temperatures after reheating, respectively, and $\xi_{R} \equiv T_{\mathrm{dark}}^{R} / T_{\mathrm{SM}}^{R}$. In figure 7 , we assume for simplicity that the dark and SM sectors reheat to the same temperature, $\xi_{R}=1$, above the weak scale. For our dark sector this leads to $T_{\text {dark }}<T_{\mathrm{SM}}$ at freeze-out, such that current $N_{\text {eff }}$ constraints are naturally evaded. We defer a more detailed discussion of decoupled dark sectors to section 3.2.

\section{Annihilations to dark sector particles}

In this section we complete the toy model of section 2.2 by explicitly introducing a coupling to radiation. We require the coupling to be large enough that DM is in kinetic equilibrium with the radiation until after freeze-out, which ensures that $T_{\text {dark }} \sim a^{-1}$. This scaling was assumed when deriving the relic density in section 2.1. If we relax the assumption that DM is in thermal contact with radiation, then the dark sector will undergo a phase of cannibalism [18, 28, 68-74], where $T_{\text {dark }}$ drops only logarithmically with the scale factor. We leave the study of coannihilation in a cannibalizing sector for future work.

There are two distinct possibilities for how DM may couple to radiation. The dark sector may couple directly to the SM, or the dark sector may contain its own light degrees 

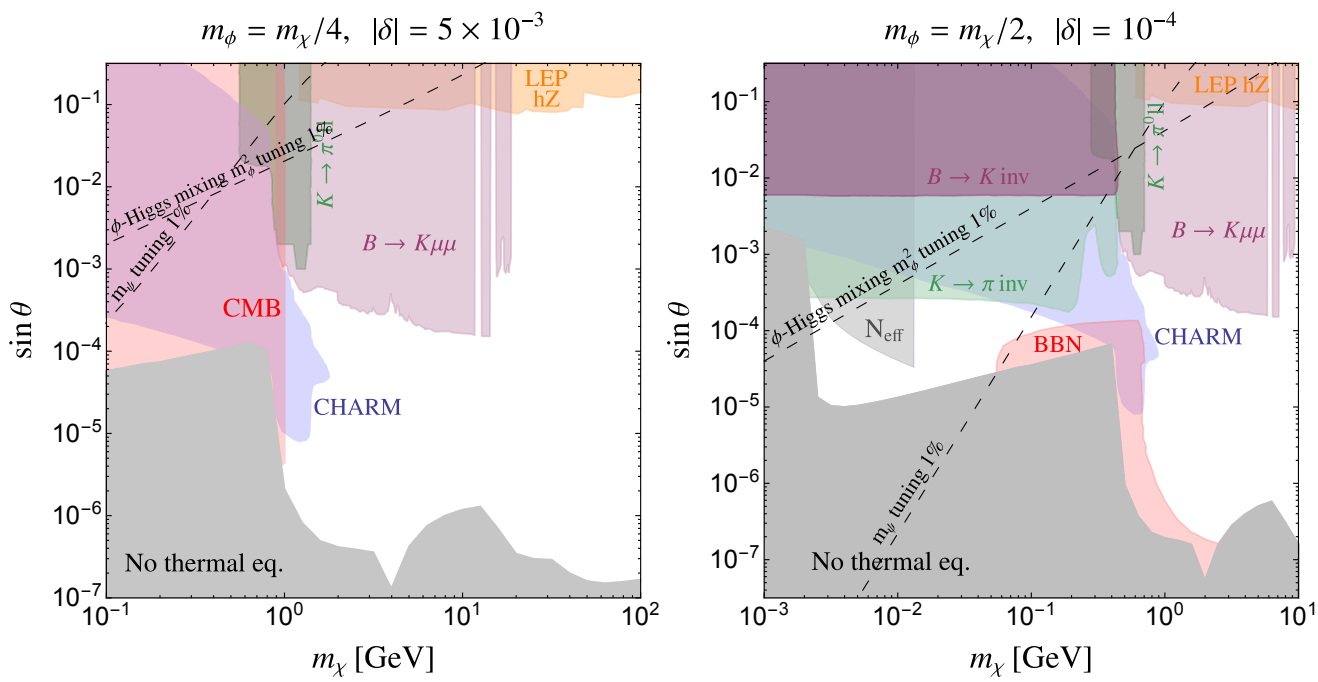

Figure 8. Phenomenology of a dark sector weakly coupled to the SM. Both panels show current constraints as a function of the DM mass, $m_{\chi}$, and the size of the mixing between the dark sector scalar and the SM Higgs boson, $\sin \theta$. The left panel shows the effect of a lighter mediator, $\phi$, and larger DM mixing, $\delta$, compared to the heavier and more weakly coupled case in the right panel. The gray shaded area at the bottom of the plot covers values of the mixing for which the dark sector is not in equilibrium with the SM at freeze-out. The other bounds are discussed in the text and come from CMB measurements from Planck [64], BBN [77, 78], meson decays [79-86], CHARM [87], and LEP $[88,89]$. In every point of the plot $\Delta$ is fixed to reproduce the observed relic density. The remaining parameters are set to $y=e^{i \pi / 4}, m_{\phi}=m_{\chi} / 4\left(m_{\chi} / 2\right)$, and $\delta=5 \times 10^{-3} e^{i \pi / 4}\left(10^{-4} e^{i \pi / 4}\right)$ in the left (right) panel.

of freedom that act as dark radiation. In the first case, constraints on $N_{\text {eff }}$ do not allow for DM lighter than the MeV scale, while a completely decoupled dark sector can have DM as light as the keV scale. As we discuss below, sub-keV masses are excluded by limits on warm DM from Lyman- $\alpha$ measurements.

We consider a dark sector that is the same as the toy model introduced in the previous section, for both the coupling to SM and dark radiation. We have two Majorana fermions $\chi$ and $\psi$, and one real scalar $\phi$ interacting through the potential of eq. (2.11). We take $m_{\psi} \gtrsim m_{\chi}>m_{\phi}$, with $\chi$ very weakly interacting through mixing of size $|\delta|=\left|\delta m / m_{\chi}\right| \ll 1$. As above we define $\Delta \equiv\left(m_{\psi}-m_{\chi}\right) / m_{\chi}$. Because we will always take the limit of very small mixing, $\delta \ll 1$, the mass eigenstates are approximately flavor eigenstates: $\chi_{1} \approx \chi$ and $\chi_{2} \approx \psi$. In the following we will abuse notation, for simplicity, and refer to the mass eigenstates also as $\chi$ and $\psi$.

\subsection{Higgs portal}

We first consider the case in which the dark sector couples to the SM through the relevant Higgs portal coupling $a_{\phi} \phi|H|^{2}$,

$$
V \supset a_{\phi} \phi|H|^{2}+\frac{\mu_{\phi}^{2}}{2} \phi^{2}+\frac{\lambda_{\phi}}{4 !} \phi^{4}-\frac{m_{H}^{2}}{2}|H|^{2}+\frac{\lambda}{4}|H|^{4} .
$$



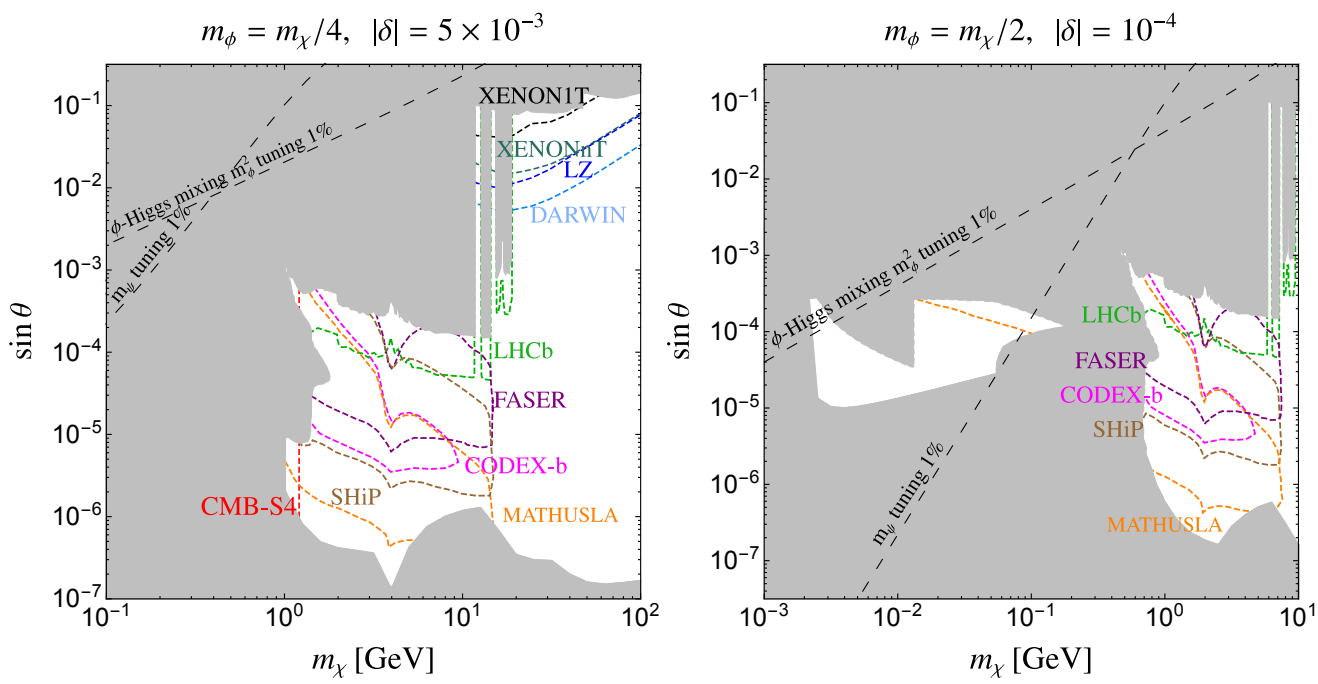

Figure 9. Phenomenology of a dark sector weakly coupled to the SM. Both panels show the projected reach of future experiments as a function of the approximate DM mass, $m_{\chi}$, and the mixing between the dark sector scalar and the SM Higgs boson, $\sin \theta$. The gray shaded area summarizes current constraints, presented separately in figure 8 . The reach for direct detection experiments [90-93], LHC-b [94], CODEX-b [94] as derived in [95], FASER [76, 96], MATHUSLA [97, 98], SHiP [99, 100] as derived in [98], and CMB Stage-4 (CMS-S4) [101] is shown. In every point of the plot $\Delta$ is fixed to reproduce the observed relic density. The remaining parameters are set to $y=e^{i \pi / 4}, m_{\phi}=m_{\chi} / 4\left(m_{\chi} / 2\right)$, and $\delta=5 \times 10^{-3} e^{i \pi / 4}\left(10^{-4} e^{i \pi / 4}\right)$ in the left (right) panel.

After electroweak symmetry breaking, we can parametrize the strength of the portal coupling using the mixing angle between $\phi$ and the SM Higgs boson

$$
\tan 2 \theta=\frac{4 a_{\phi} v}{\lambda v^{2}-\lambda_{\phi} v_{\phi}^{2}-2 \mu_{\phi}^{2}},
$$

where $v \approx 246 \mathrm{GeV}$ and $v_{\phi} \equiv\langle\phi\rangle$ is the VEV of $\phi$.

The decay width and branching fractions of $\phi$ are crucial for determining both the experimental prospects and when the SM and dark sector are in thermal equilibrium. We take the scalar $\phi$ to be lighter than DM, such that it can only decay to SM particles. We note that the width and branching fractions of $\phi$ cannot be determined with precision when its mass is between $\sim 2 m_{\pi}$ and a few $\mathrm{GeV}$, due to significant hadronic uncertainties [75]. For the width and branching fractions, we follow refs. [76].

The phenomenology of the model is summarized in figures. 8 and 9. These figures show $m_{\chi}$ versus the singlet-Higgs mixing, $\sin \theta$, with $\Delta$ chosen at each point so that the relic density matches observation. figure 8 shows current constraints, while figure 9 presents the projected reach of future experiments. There is a lower bound on $\sin \theta$ set by the requirement that $\phi$ interactions with the SM are efficient at keeping the two sectors in thermal equilibrium at DM freeze-out. Within the dark gray shaded areas at the bottom of figure 8, the two sectors are not in equilibrium when DM freezes-out. Thermalization can be achieved by rapid $\phi$ decay or $2 \rightarrow 2$ scattering processes. We find that $\phi$ decay is more efficient for our choice of parameters. The CMB constraints on $N_{\text {eff }}$ and on the DM 
annihilation cross section [64] in figure 8 set a lower bound on $m_{\chi}$, almost independent of the size of the $\phi$-Higgs mixing angle. These two constraints are common to all realizations of coannihilation coupled to the SM, and we now describe them in more detail.

The CMB bound on $N_{\text {eff }}$ excludes $\chi$ masses below $\sim 10 \mathrm{MeV}$. If the dark sector is in equilibrium with photons after neutrinos decouple, then DM transfers entropy to the photon bath, increasing the photon temperature relative to neutrinos [102]. This is a familiar effect in the SM, where photons are heated relative to neutrinos after electrons turn non-relativistic. Here, DM lowers $N_{\text {eff }}$, since neutrinos are cooled relative to the photon temperature. There is one important subtlety: if $\sin \theta$ is sufficiently small, the dark sector may not be in kinetic equilibrium with the SM at neutrino decoupling, although it may later equilibrate before DM annihilations decouple [103]. In this region, the value of $N_{\text {eff }}$ probed by the CMB is sensitive to initial conditions. We can always choose a low enough reheating temperature, compatible with BBN, for which there is no constraint on $N_{\text {eff }}$. For this reason, we do not plot the $N_{\text {eff }}$ bound in this part of the parameter space, as can be seen in the right panel of figure 8 , where the light gray shaded region ends at $\sin \theta \approx 10^{-4}-10^{-5}$. We do not plot this constraint in the left panel of figure 8 since it is everywhere weaker than the CMB bound on DM annihilations.

DM annihilations, $\chi \chi \rightarrow \phi \phi$, with subsequent $\phi$ decay to SM particles, can alter the recombination history through energy injection. In particular, electromagnetically interacting products in the annihilation cascade can cause ionization, heating, and excitation of hydrogen during the cosmic dark ages, leading to observable modifications of the CMB anisotropies [104, 105]. The rate of energy release per unit volume is proportional to $f_{\text {eff }}\langle\sigma v\rangle / m_{\mathrm{DM}}$, becoming larger for light thermal relics, which are excluded below about $10 \mathrm{GeV}$, depending on the annihilation products [64, 106, 107]. (A notable exception is DM annihilations to neutrinos, which are not strongly constrained by the CMB [108, 109].) In our model, the strongly interacting heavier state, $\psi$, decays down to DM, $\chi$, before recombination. The CMB is only sensitive to DM self-annihilations, which have cross section suppressed by small $\delta$. Therefore, much lighter DM masses are compatible with CMB annihilations in our model compared to WIMPs. The efficiency factor, $f_{\text {eff }}$, measures the fraction of the DM mass that is converted to heating the photon-baryon plasma, and captures the model dependence of the DM annihilation products. We follow the prescription from [110] to calculate $f_{\text {eff }}$ for the cascade annihilation and use $f_{\text {eff }}$ tables provided in [106]. The corresponding Planck bound [64] is shown in the left panel of figure 8 as a red shaded area. In figure 9, we show the projected improvement of a factor of 3 in cross section reach from CMB Stage-4, assuming $60 \%$ sky coverage [101]. In the right panel of figure 8 , we choose a smaller value of $\delta$ where the bound is completely absent.

An additional constraint comes from BBN and applies when $\phi$ is heavier, as in right panel of figure 8 (red shaded area). As $\psi$ becomes heavier, the splitting, $\Delta$, that matches the observed relic density becomes smaller. When the DM mass is greater than about $100 \mathrm{MeV}$, $\psi$ can only decay through an off-shell $\phi$ to $\chi$ plus SM states. For small mixing angle $\theta$, the $\psi$ lifetime can become long enough to affect BBN through injection of electromagnetic particles into the primordial plasma [77, 78]. When the mass splitting between $\psi$ and $\chi$ is larger than $2 m_{\pi}$, the dominant decay channels have mesons in the final state. However, 
we find that the strongest bound comes from the electromagnetic fraction of these decays, because the abundance of $\psi$ is too small to affect proton-neutron conversions at early stages of BBN [111], and the injected hadrons are not energetic enough for hadro-dissociation to affect primordial light element abundances $[112,113]$.

A variety of other observations are also relevant to our parameter space. Constraints from meson decays $[75,79-86,114,115]$ and colliders $[88,89,116]$ follow directly from $\phi$ 's interactions with the SM, and are unrelated to DM. In our model these constraints play a role at large mixing, $\sin \theta \gtrsim 10^{-3}$, as shown in figure 8 . The CHARM proton beam dump searched for long-lived particles [87], and excludes part of the parameter space of a scalar mixed with the Higgs $[75,99,117]$. In this case, the scalar $\phi$ can be produced from meson decays, and can be detected when it decays within the displaced detector.

The most promising avenues for probing more parameter space are DM direct detection [90-93], as can be seen in the left panel of figure 9, and future beam dump experiments such as SHiP [99, 100]. Experiments exploiting the abundance of mesons in LHC collisions, such as CODEX-b [94], MATHUSLA [97, 98], and FASER [76, 96] are competitive with SHiP. In figure 9 we also show the projected reach for LHCb as derived in ref. [94]. The reach of these experiments depends on the type of portal that connects the SM to the dark sector and the size of the portal coupling. However, we note that any realization of light DM from coannihilation, that is coupled to the SM, predicts the presence of new light particles that can be searched for in intensity frontier experiments [65, 118-121].

To illustrate the phenomenology of the model in figure 8 we have chosen two representative slices of parameter space. For those choices of $\delta$ and $m_{\phi}$ we are in the sterile coannihilation regime outside of the gray shaded area. Note however that at smaller $\delta$ the relic density becomes again dependent on the dark matter couplings, since around $\delta \approx 10^{-5}\left(10^{-6}\right)$ in the left (right) panel we enter the coscattering regime.

To conclude this section, it is appropriate to mention that this model has several potential sources of tuning. There is a hierarchy problem for $\phi$, as a consequence of its $\mathcal{O}(1)$ Yukawa coupling to $\psi$ (analogous to the top quark coupling to the SM Higgs). Therefore the $\phi$ mass has tuning of size $\sim y^{2} /(4 \pi)^{2} \times m_{\phi}^{2} / \Lambda^{2}$, where $\Lambda$ is the high-energy cutoff of the model. This tuning is naturally removed if the model is UV-completed to be supersymmetric or to have $\phi$ as a composite state. We leave the exploration of such UV completions for future work. There is also tuning of the $\phi$ mass due to its mixing with the Higgs boson, because the $\phi$-Higgs mass matrix has an off-diagonal term of size $a_{\phi} v$. This tuning has parametric size $\sim m_{\phi}^{2} /\left[\lambda v^{2}(\tan 2 \theta)^{2}\right]$ and is worse than $1 \%$ above the dashed line in figures. 8 and 9. This tuning is not independent of the $\phi$ hierarchy problem, since one tuning is needed to keep $\phi$ light. We highlight this region since it is difficult to remove the tuning from singlet-Higgs mixing using a UV completion, because the tuning follows from IR parameters. We note that the tuning from $\phi$-Higgs mixing applies generically to theories with a light scalar mixing with the Higgs [75, 95, 122], since it does not depend on the Yukawa coupling of $\phi$ to $\psi$.

There is also a second potential tuning required to keep $\psi$ light, since $m_{\psi}$ gets a contribution from the VEV of $\phi$ (which is unavoidable because a tadpole is generated for $\phi$ after electroweak symmetry breaking). This tuning has parametric size 


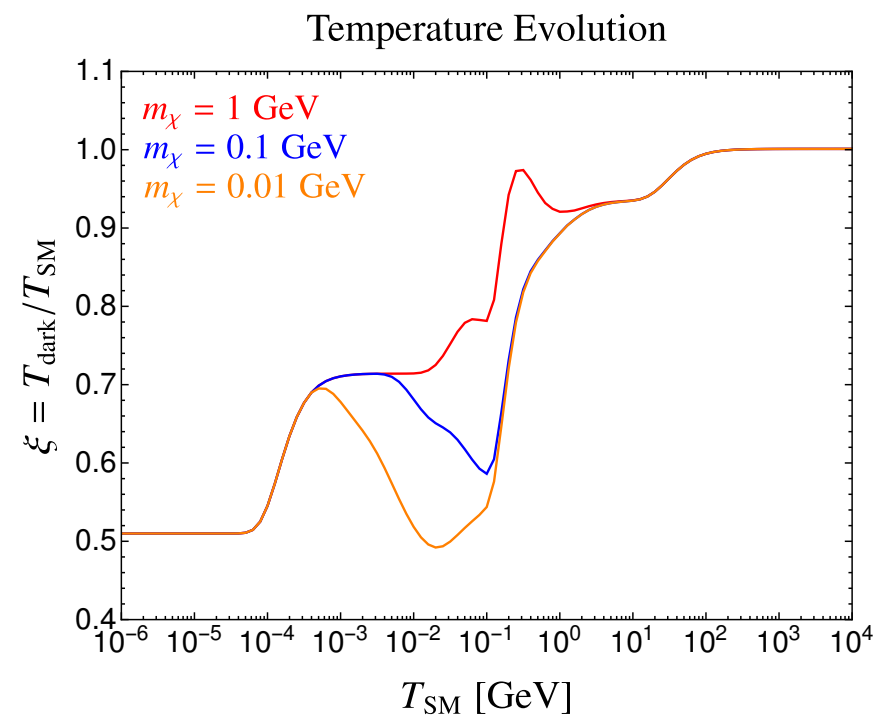

Figure 10. Dark sector temperature evolution as a function of the SM photon temperature in the decoupled dark sector model of section 3.2. We have assumed that the two sectors begin at the same temperature, $\xi_{R}=1$, above the weak scale. For each $m_{\chi}, \Delta$ is fixed to reproduce the observed relic density. The remaining parameters are set to $m_{\phi}=m_{\chi} / 4, y=e^{i \pi / 4}$, and $\delta=10^{-4} e^{i \pi / 4}$.

$\left[\lambda_{\phi} /(\lambda \tan 2 \theta)\right]^{1 / 3} m_{\psi} /(y v)$, and is worse than $1 \%$ above the second dashed line in figures 8 and 9 .

\subsection{Decoupled dark sector}

Sterile coannihilation can also be realized in a completely decoupled sector. In this case, the dark sector has its own temperature which can be colder than the SM sector, allowing the DM mass to drop below the $\mathrm{MeV}$ scale consistent with bounds from $N_{\text {eff }}$. As an example we consider again the model described at the beginning of section 2.2, supplemented with a new Majorana fermion $n$ that acts as dark radiation. We note that our calculation of the relic density has assumed that DM is in thermal equilibrium with some form of radiation, and therefore dark radiation is required if the dark sector is decoupled from the SM.

A simple potential that keeps DM in thermal equilibrium with the dark radiation, $n$, is

$$
V \supset \frac{m_{n}}{2} n^{2}+\frac{y_{n}}{2} \phi n^{2} .
$$

In what follows we take $m_{n} \ll 1 \mathrm{eV}$, which ensures that $\Omega_{n} / \Omega_{\mathrm{DM}} \ll 10 \%$. We fix $y_{n}=10^{-4}$, which keeps $n$ in equilibrium with the dark sector. The reheating is captured by the parameter $\xi_{R}=T_{\text {dark }}^{R} / T_{\mathrm{SM}}^{R}$, where $T_{\text {dark }, S M}^{R}$ are the temperatures of the two sectors at reheating. In what follows, we assume that the SM is reheated above the electroweak scale, $T_{\mathrm{SM}}^{R}>250 \mathrm{GeV}$. The relative temperatures of the two sectors, $\xi=T_{\text {dark }} / T_{\mathrm{SM}}$, evolves following conservation of relative entropies of the two sectors (eq. (2.13)), and depends on the number of relativistic degrees of freedom in each sector. Figure 10 shows the temperature evolution, assuming $\xi_{R}=1$, for a few representative dark spectra. We see that the dark sector ends up cooler than the SM, when it starts with the same temperature, as a consequence of the larger number of degrees of freedom in the SM sector. 

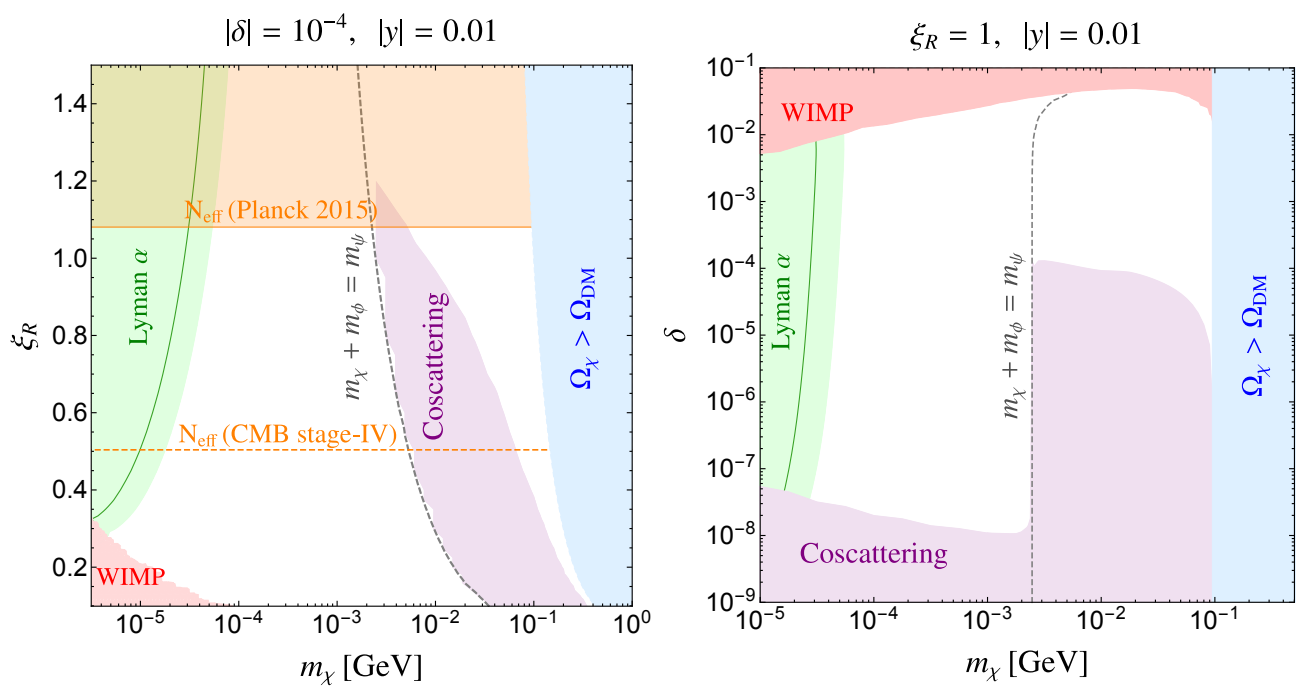

Figure 11. Phenomenology of a dark sector decoupled from the SM, but in equilibrium with dark radiation. In the left panel we show the bound on the relative temperatures of the dark and SM sectors at reheating, $\xi_{R}=T_{\mathrm{dark}}^{R} / T_{\mathrm{SM}}^{R}$, as a function of the DM mass. We show constraints on $N_{\text {eff }}$ from current Planck measurements [64] and the projected CMB Stage-4 sensitivity [101]. In the right panel we show the DM mixing angle, $\delta$, with the active state, versus the DM mass. When the mixing is too large or small the relic density is no longer set by coannihilation. The range of allowed DM masses is determined by DM overproduction and constraints on the free-streaming length from Lyman- $\alpha$ measurements [139-142]. In every point of the plot, $\Delta$ is fixed to reproduce the observed relic density. The remaining parameters are set to $y=0.01 e^{i \pi / 4}, m_{\phi}=m_{\chi} / 4$, and $\delta=10^{-4} e^{i \pi / 4}$ $\left(\xi_{R}=1\right)$ in the left (right) panel.

Even if only gravitationally coupled to the SM, there are several ways that the dark sector can be probed observationally. First, $N_{\text {eff }}$ during BBN or the CMB epoch can be modified. Second, if the DM mass is sufficiently small, the matter power spectrum can deviate from the $\Lambda$ CDM prediction [64, 123-131]. Third, DM self-interactions can leave imprints on astrophysical observations [132-138]. However, in sterile coannihilation, selfinteractions are generically suppressed by the small couplings ( $\delta$ in our case) of DM to the mediator. In our model, the DM self-interaction cross section is smaller than weak scale, whereas observable self-interactions requires a nuclear sized cross section.

In the left panel of figure 11 we show the current bound from $N_{\text {eff }}$, as measured by Planck [64], on the reheating temperature of the dark sector. A dark sector reheated slightly above the SM is still consistent with observations. We also display the projected sensitivity of CMB Stage-4 [101]. BBN bounds on $N_{\text {eff }}$ are subleading.

In the decoupled dark sector, the smallest DM mass consistent with observations is determined by Lyman- $\alpha$ measurements [139-142]. Two effects can suppress the matter power spectrum: the free-streaming of $\mathrm{DM}[130,131]$ and dark acoustic oscillations [131, 143-145]. We find that the second effect is subdominant in our parameter space, and the lower bound on the DM mass is set by the free-streaming length.

After decoupling from dark radiation, DM particles stream freely due to their nonzero velocity dispersion, damping density perturbations below a certain scale. This free- 
streaming scale is given by the comoving length scale that a DM particle can travel between the time of kinetic decoupling and matter radiation equality. DM is kept in kinetic equilibrium with dark radiation through (inverse-)decay, $\psi \leftrightarrow \chi \phi$, where $\phi$ is in thermal contact with dark radiation. For parameters where the free streaming bound is relevant, we find that decays decouple later than scatterings (such as $\chi \phi \rightarrow \chi \phi$ ). We estimate the temperature of the SM bath at DM kinetic decoupling, $T_{\mathrm{SM}}^{\mathrm{kd}}$, using the relation $m_{\psi} \Gamma_{\psi}\left[n_{\psi} /\left(\rho_{\chi}+\rho_{\psi}\right)\right]_{T_{\mathrm{SM}}^{\mathrm{kd}}}=H\left(T_{\mathrm{SM}}^{\mathrm{kd}}\right)$ (see for example ref. [146]). The free-streaming length is

$$
\begin{aligned}
\lambda_{\mathrm{FS}} & =\int_{t_{\mathrm{kd}}}^{t_{\mathrm{EQ}}} \frac{v(t)}{a(t)} d t \\
& \approx \frac{1}{a\left(T_{\mathrm{EQ}}\right)} v_{\mathrm{kd}} \frac{3 \sqrt{5} M_{\mathrm{Pl}}}{2 \pi^{3 / 2} g_{*}\left(T_{\mathrm{EQ}}\right)^{1 / 2} T_{\mathrm{EQ}} T_{\mathrm{SM}}^{\mathrm{kd}}} \log \left(\frac{T_{\mathrm{SM}}^{\mathrm{kd}}}{T_{\mathrm{EQ}}}\right) \\
& \approx 0.124 \mathrm{Mpc} v_{\mathrm{kd}} \frac{\mathrm{keV}}{T_{\mathrm{SM}}^{\mathrm{kd}}} \log \left(\frac{1.4 T_{\mathrm{SM}}^{\mathrm{kd}}}{\mathrm{eV}}\right),
\end{aligned}
$$

where $v_{\mathrm{kd}} \sim \sqrt{T_{\mathrm{dark}}^{\mathrm{kd}} / m_{\chi}}$ is the velocity of DM particles at kinetic decoupling, $T_{\mathrm{EQ}}$ is the SM temperature at matter radiation equality, and we have approximated the number of relativistic degrees of freedom, $g_{*}$, as constant between $T_{\mathrm{SM}}^{\mathrm{kd}}$ and $T_{\mathrm{EQ}}$.

In figure 11, we show the bound for $\lambda_{\mathrm{FS}} \gtrsim 0.06 \mathrm{Mpc}$ [141] (green shaded area) and $\lambda_{\mathrm{FS}} \gtrsim 0.1 \mathrm{Mpc}[139]$ (green line). When the dark sector is reheated to the same temperature as the SM, DM masses below $\sim 100 \mathrm{keV}$ are excluded. The bound becomes weaker if the dark sector is colder than the SM, as shown in the left panel of figure 11, and we find viable sterile coannihilation models with the DM as light as $m_{\chi} \approx 5 \mathrm{keV}$. We find that the constraint from Lyman- $\alpha$ is stronger than the Tremaine-Gunn bound [147], which sets a lower limit on the mass of fermionic DM of several hundred eV [148].

The rest of our parameter space is bounded by regions where coannihilation is not setting the relic density. At large $\delta$ or small $\xi_{R}, \chi \chi$ annihilations dominate freeze-out and DM becomes WIMP-like. When $\delta$ is too small, processes that exchange $\chi$ and $\psi$ decouple before annihilations, and freeze-out enters the coscattering phase [15]. Finally, at large $m_{\chi}$ (and fixed $y$ ), the relic density is too large because the effective annihilation cross section, which scales as $y^{4} / m_{\chi}^{2}$, becomes smaller than weak scale for any choice of $\Delta$.

\section{Direct annihilations to standard model particles}

Coannihilations consisting of direct annihilations to SM particles offer unique detection prospects in future experiments. Existing studies have highlighted thermal targets, where masses and couplings are chosen such that the relic density matches the observed abundance of DM. Several studies have considered coannihilation, but focus on models where DM participates in the dominant annihilation channel $[14,61,63,149]$. In these models, the lifetime of heavy states to decay to DM is related to the DM relic density, providing predictive targets for future experiments that probe this lifetime.

In this section, we highlight the sterile coannihilation regime, where the heavy state annihilates directly to the SM. The relic density is independent of the DM couplings, since 

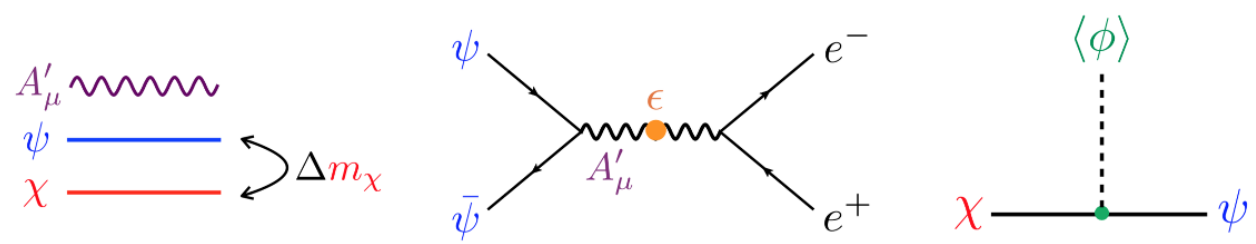

Figure 12. Schematic representation of the model described in section 4. From left to right: mass spectrum, main annihilation channel, and mixing between DM, $\chi$, and its coannihilating partner, $\psi$.

DM does not participate in the dominant annihilations. Therefore, sterile coannihilation points to a broad parameter space of thermal targets, where the lifetime of the heavy states can be varied without changing the relic density. If the lightest state is DM today, the only robust requirement is that the lifetime of the heavy states should be shorter than the present age of the Universe.

We illustrate this point using the following model: the dark sector contains two Weyl spinors, $\psi$ and $\psi^{c}$, charged under a dark gauge group, $\mathrm{U}(1)_{D}$, with gauge boson $A_{\mu}^{\prime}$ and gauge coupling $g_{D} \equiv \sqrt{4 \pi \alpha_{D}}$. The gauge symmetry is spontaneously broken by a complex scalar $\phi$ with VEV $\langle\phi\rangle \equiv v_{\phi}$. Gauge charges are +1 for $\psi$ and -1 for $\phi$ and $\psi^{c}$. We include an additional neutral Majorana spinor, $\chi$, which is our DM candidate. The fermionic part of the dark sector potential is

$$
V \supset m_{\psi} \psi^{c} \psi+\frac{m_{\chi}}{2} \chi^{2}+y \phi \psi \chi+\bar{y} \phi^{\dagger} \psi^{c} \chi+\text { h.c. },
$$

where all parameters $\left(m_{\psi}, m_{\chi}, y\right.$, and $\left.\bar{y}\right)$ are complex. We can remove 3 phases, by redefining the fields, so we are left with 4 real parameters and 1 phase. We consider the sterile coannihilation limit, $y v_{\phi} / m_{\psi, \chi} \ll 1$ and $\bar{y} v_{\phi} / m_{\psi, \chi} \ll 1$, such that $\chi$ corresponds to DM, while $\psi$ and $\psi^{c}$ correspond to the heavy states whose annihilations dominate the effective cross section of eq. (2.3). In this example, we take $m_{\psi} \geq m_{\chi}$ and define $\Delta \equiv$ $\left(m_{\psi}-m_{\chi}\right) / m_{\chi}$ as the approximate mass splitting between DM and its two coannihilating partners (that are almost degenerate). We assume that $m_{\phi}>m_{\chi, \psi}$, so that annihilations into $\phi$ can be neglected.

We consider the regime $m_{A^{\prime}}>m_{\psi, \chi}$, and we assume that there is a kinetic mixing between the new gauge group $\mathrm{U}(1)_{D}$ and electromagnetism

$$
\mathcal{L} \supset-\frac{\epsilon}{2} F_{d}^{\mu \nu} F_{\mu \nu},
$$

where $F_{d}$ is the field strength of the dark gauge force and $\epsilon$ sets the strength of the mixing.

The spectrum contains three fermionic mass eigenstates $n_{1,2,3}$, which we order by ascending mass $\left(m_{n_{i}}<m_{n_{j}}\right.$ if $\left.i<j\right) . n_{1}$ is mostly $\chi$ and is weakly interacting and $n_{2,3}$ are mostly made up of $\psi$ and $\psi^{c \dagger}$. The main ingredients of the model are summarized in figure 12. As shown in the figure, coannihilation proceeds through an off-shell dark photon into SM particles. Kinetic equilibrium with the SM is insured by dark photon decays and DM scattering off SM states. The only $\mathcal{O}\left(g_{D}\right)$ coupling after mass diagonalization pairs 
up $n_{2}$ and $n_{3}$ in the dark photon vertex. The other couplings are suppressed by powers of $\delta \equiv y v_{\phi} / m_{\chi}$ and $\bar{\delta} \equiv \bar{y} v_{\phi} / m_{\chi}$. Therefore, as for the toy model in section 2.2, so long as $\delta$ and $\bar{\delta}$ are sufficiently small, the relic density is independent of them, since

$$
\left\langle\sigma_{\mathrm{eff}} v\right\rangle=\left\langle\sigma_{23} v\right\rangle+\mathcal{O}\left(\delta^{2}, \bar{\delta}^{2}\right)
$$

where $\sigma_{23}$ is the annihilation cross section of $n_{2} n_{3}$ to SM states. As shown in figure 13 this is true for many orders of magnitude in $\delta$ and $\bar{\delta}$, but when the dark matter interactions become too small we enter the coscattering regime.

The phenomenology of the model strongly depends on $\delta$ and $\bar{\delta}$, for two reasons. First, $n_{1}$ interactions are suppressed by $\delta$ and $\bar{\delta}$, and in most of the parameter space DM today is entirely composed of this lightest state. Second, the heavier and more strongly interacting fermions, $n_{2,3}$, have decay widths proportional to these small parameters, $\Gamma_{n_{2,3}} \sim \delta^{2}, \bar{\delta}^{2}$.

In the laboratory, $n_{2,3}$ are the states that are dominantly produced, and their decay length, into $n_{1}$, determines whether they can be detected or not. A quantitative illustration of this point is presented in figure 13. There we fix the dark sector mass scale, $m_{\chi}$, and dark gauge coupling $\alpha_{D}$, and look at the reach on the dark photon coupling to the SM, $\epsilon$, as a function of the parameter $\delta$. For simplicity we take $\bar{\delta}=\delta / 2$. At every point $\Delta$ is chosen to reproduce the observed relic density. ${ }^{1}$

We show in figure 13 the bound from the LSND [153] proton beam dump and the projected reach of the BDX [160] and MiniBooNe [161, 162] electron beam dumps. The sensitivity of these experiments comes from the production of the dark photon, $A^{\prime}$, which subsequently decays promptly with a nearly $100 \%$ branching ratio to $n_{2} n_{3}$. Then if at least one of the two states decays within the active volume of the detector, the leptons from $n_{2,3} \rightarrow e^{+} e^{-} n_{1}$ can be detected. The reach in $\epsilon$ of these three beam dumps depends on the lifetime of $n_{2}$ and $n_{3}$, and therefore on $\delta$. To derive the bounds and reach, we used the results of ref. [61], after accounting for the different lifetime of the excited state in our model. We include a factor of 2 enhancement to our signal rate, compared to ref. [61], because each dark photon decay produces two excited states.

We find that the proposed LDMX electron beam dump [158] can probe the entire allowed region of the left panel of figure 13. In our model the LDMX signal will be missing energy events, from the production of an $A^{\prime}$, followed by its decay to $n_{2} n_{3}$ (surviving past the detector calorimeters). In the right panel of figure 13, the dark photon is too heavy to be produced at LDMX so there is no sensitivity.

Even after fixing the relic density, the lifetime of $n_{2}$ and $n_{3}$ can be large enough that decays during BBN [77, 78] and during recombination [154] become relevant. Decays of the heavier states can also leave CMB spectral distortions detectable by the proposed PIXIE satellite [154-157]. Spectral distortions are a generic signal of sterile coannihilation,

\footnotetext{
${ }^{1}$ For the DM mass chosen in the right panel of figure 13, DM annihilations into hadronic final states play an important role and are not treated by MicrOMEGASv4, which works in the partonic limit. In this case, we solve the Boltzmann equation numerically, and we take into account annihilation into hadrons by replacing the annihilation cross section into leptonic final states with $(\sigma v)_{e^{+} e^{-}}+(\sigma v)_{\mu^{+} \mu^{-}}[1+R(s)]$, where $(\sigma v)_{e^{+} e^{-}}$and $(\sigma v)_{\mu^{+} \mu^{-}}$are the annihilation cross sections of DM into $e^{+} e^{-}$and $\mu^{+} \mu^{-}$respectively and $R(s)$ is the $R$-ratio for $e^{+} e^{-}$hadronic annihilation. We use the $R(s)$ values provided by the PDG [159].
} 

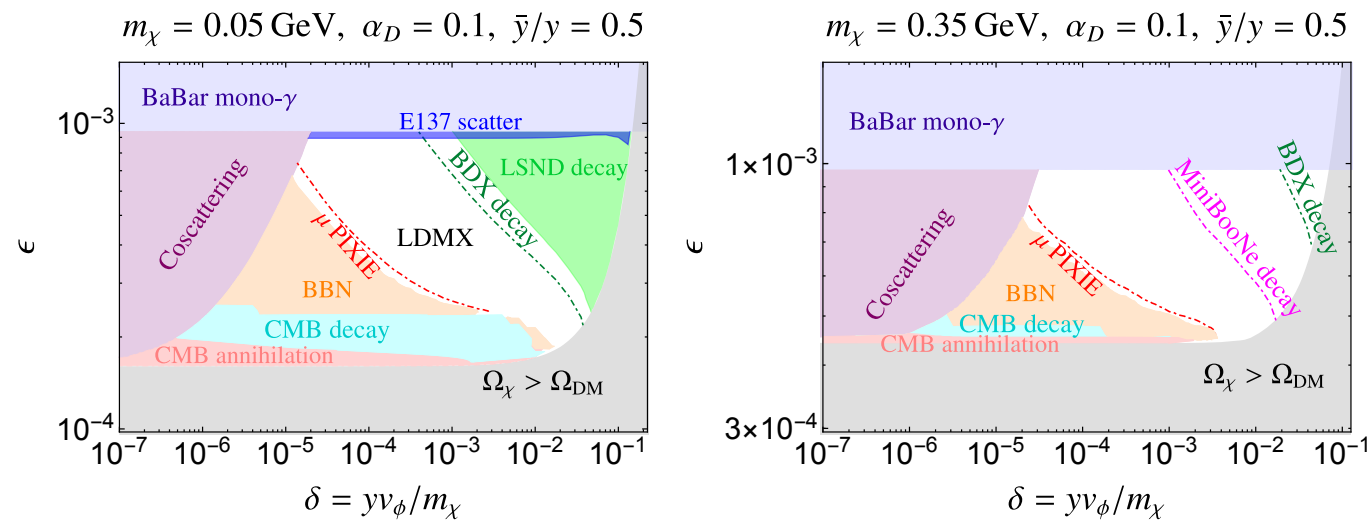

Figure 13. Dark photon coupling, $\epsilon$, vs. the size of the DM mixing with its coannihilating states, $\delta$. When $\delta$ is sufficiently small the relic density does not depend on it, as shown by the flat $\Omega_{\chi}>\Omega_{\mathrm{DM}}$ contour. On the contrary the lifetime of the heavier states, sharing a conserved quantum number with DM, increases as $\delta^{2}$, strongly affecting the phenomenology. The bounds are discussed in the text and come from BaBar [150, 151], E137 [152], LSND [153], BBN [77, 78], and the CMB [154]. The reach for BDX and MiniBooNe adapted from [61], and PIXIE [154-157] (corresponding to $\mu<5 \times 10^{-8}$ ) are shown. LDMX [158] can probe the entire allowed region of the left panel, but is not sensitive to the heavier dark photon mass on the right panel. In every point of the plot $\Delta$ is fixed to reproduce the observed relic density. The remaining parameters are set to $m_{\chi}=0.05(0.35) \mathrm{GeV}$, $m_{A^{\prime}}=3 m_{\chi}(1+\Delta), \alpha_{D}=0.1$, and $\bar{y} / y=0.5$ in the left (right) panel.

because the heavier coannihilating states have a long lifetime in a large fraction of the parameter space. This is due to the insensitivity of the relic density to the small mixing between DM and the active states. We note that a signal in LDMX, plus the detection of spectral distortions, would point to DM from sterile coannihilation.

In the region where $\Delta$ approaches zero, $n_{2}$ and $n_{3}$ can live long enough that they are still present at the time of recombination. When this happens, their annihilations can alter the CMB temperature and polarization power spectra. We show the corresponding bound from Planck [64] in figure 13.

The remaining constraints that we show in figure 13 are a monophoton search from BaBar [150, 151], and the electron beam dump E137 [152]. In the latter experiment, $n_{2}$ and $n_{3}$ are produced through an on-shell dark photon, as in the previous cases, and detected through scattering off electrons $n_{2,3} e^{-} \rightarrow n_{3,2} e^{-}$.

We have seen that sterile coannihilation, with annihilations of the heavy state directly into the SM, has a rich phenomenology. Sterile coannihilation is generically described by a weakly interacting DM candidate accompanied by heavier, more strongly interacting, states that can have cosmologically long lifetimes. Sterile coannihilation leads to a broader parameter space of lifetimes, consistent with the observed DM abundance, than the thermal targets of non-sterile coannihilating models.

\section{Conclusions}

In this paper, we discussed how coannihilation constitutes a novel mechanism for light DM that evades CMB constraints on WIMPs. Traditionally, coannihilation has been applied 
to weak scale DM, where it is only relevant for highly degenerate states. As we have emphasized, non-degenerate coannihilation naturally realizes light DM. This is because the annihilation rate of heavy states is exponentially suppressed, by their equilibrium number density, when computing the effective annihilation rate that dictates the DM relic density (see eq. (2.3)). If the annihilations of heavy states dominate, they must have a cross section that is exponentially larger than the weak scale, in order to overcome this exponential suppression. To generate a large cross section, the mass scale of the dark states should be exponentially lighter than the weak scale. The CMB bound is evaded simply because the heavy states can decay to DM before recombination, such that the annihilations that dictate the relic density stop occurring before the CMB forms.

We have focused on the sterile coannihilation limit, where DM does not participate in the dominant annihilations. The relic density depends on the DM mass, but not its couplings. This is a counterexample to the lore that the DM's couplings are determined by its abundance, once its mass is specified. Therefore, sterile coannihilation leads to a broader parameter space of thermal targets for future experiments than is usually considered.

Light DM from coannihilation is easily realized in a variety of dark sectors. We have studied three example models, where mostly sterile DM mixes with a heavier state that experiences rapid annihilations. In section 3.2, we considered the case that the dark sector contains dark radiation and is totally decoupled from the SM sector. We found that the DM can be as light as the keV scale. In section 3.1, we considered DM that is coupled to the SM and annihilates into a dark Higgs that mixes with the SM Higgs. This possibility is more minimal than the decoupled scenario, because it does not require dark radiation and does not depend on the initial DM temperature. The lower bound on the DM mass is raised by three orders of magnitude, compared to the decoupled case, due to constrains on $N_{\text {eff }}$ from BBN and the CMB. We identified rich experimental prospects for production and detection of the dark states. In section 4 , we considered direct annihilations to the SM in the limit of sterile coannihilation. This scenario provides an explicit example of thermal targets that are independent of DM's couplings and therefore span a wide range of lifetimes for the heavy states to decay. Sterile coannihilation points to a characteristic combination of signals in future missing momentum experiments and CMB spectral distortions.

We note that light DM from coannihilation is a broad framework that extends beyond the specific example models considered in this paper. It would be interesting to look at more example dark sectors exhibiting light DM from coannihilation, and more portals that connect them to the SM. It is worthwhile to develop explicit models where the dark sector is supersymmetric, or where the DM and annihilating states are composites of a strongly coupled hidden sector, such that there masses are naturally understood. Finally, we note that coannihilation can be combined with other mechanisms, such as forbidden annihilations, dark sector cannibalism, and 3-to-2 annihilations.

\section{Acknowledgments}

The authors would like to thank Francesco D'Eramo, Daniel Egana-Ugrinovic, Yonit Hochberg, Duccio Pappadopulo, Gabriele Trevisan, Neal Weiner, and Mike Williams for 
helpful discussions. RTD is supported by the U.S. Department of Energy under Contract No. DE-AC02-76SF00515. JTR is supported by NSF CAREER grant PHY-1554858. CM would like to thank the CERN Theoretical Physics Department for hospitality while this work was completed. RTD and JTR thank the hospitality of the Aspen Center for Physics, which is supported by the NSF grant PHY-1607611.

Open Access. This article is distributed under the terms of the Creative Commons Attribution License (CC-BY 4.0), which permits any use, distribution and reproduction in any medium, provided the original author(s) and source are credited.

\section{References}

[1] B.W. Lee and S. Weinberg, Cosmological lower bound on heavy neutrino masses, Phys. Rev. Lett. 39 (1977) 165 [inSPIRE].

[2] E.W. Kolb and M.S. Turner, The Early universe, Front. Phys. 69 (1990) 1 [InSPIRE].

[3] P. Gondolo and G. Gelmini, Cosmic abundances of stable particles: Improved analysis, Nucl. Phys. B 360 (1991) 145 [inSPIRE].

[4] G. Jungman, M. Kamionkowski and K. Griest, Supersymmetric dark matter, Phys. Rept. 267 (1996) 195 [hep-ph/9506380] [INSPIRE].

[5] CRESST collaboration, G. Angloher et al., Results on light dark matter particles with a low-threshold CRESST-II detector, Eur. Phys. J. C 76 (2016) 25 [arXiv:1509.01515] [INSPIRE].

[6] SuperCDMS collaboration, R. Agnese et al., New results from the search for low-mass weakly interacting massive particles with the CDMS low ionization threshold experiment, Phys. Rev. Lett. 116 (2016) 071301 [arXiv: 1509.02448] [INSPIRE].

[7] LUX collaboration, D.S. Akerib et al., Improved limits on scattering of weakly interacting massive particles from reanalysis of 2013 LUX data, Phys. Rev. Lett. 116 (2016) 161301 [arXiv: 1512.03506] [INSPIRE].

[8] XENON collaboration, E. Aprile et al., First dark matter search results from the XENON1T experiment, Phys. Rev. Lett. 119 (2017) 181301 [arXiv:1705. 06655] [INSPIRE].

[9] PandaX-II collaboration, X. Cui et al., Dark matter results from 54-ton-day exposure of PandaX-II experiment, Phys. Rev. Lett. 119 (2017) 181302 [arXiv:1708.06917] [InSPIRE].

[10] K. Griest and D. Seckel, Three exceptions in the calculation of relic abundances, Phys. Rev. D 43 (1991) 3191 [INSPIRE].

[11] R.T. D'Agnolo and J.T. Ruderman, Light dark matter from forbidden channels, Phys. Rev. Lett. 115 (2015) 061301 [arXiv: 1505.07107] [INSPIRE].

[12] A. Delgado, A. Martin and N. Raj, Forbidden dark matter at the weak scale via the top portal, Phys. Rev. D 95 (2017) 035002 [arXiv: 1608.05345] [InSPIRE].

[13] M. Ibe, H. Murayama and T.T. Yanagida, Breit-Wigner enhancement of dark matter annihilation, Phys. Rev. D 79 (2009) 095009 [arXiv: 0812.0072] [InSPIRE].

[14] J.L. Feng and J. Smolinsky, Impact of a resonance on thermal targets for invisible dark photon searches, Phys. Rev. D 96 (2017) 095022 [arXiv:1707.03835] [InSPIRE].

[15] R.T. D'Agnolo, D. Pappadopulo and J.T. Ruderman, Fourth exception in the calculation of relic abundances, Phys. Rev. Lett. 119 (2017) 061102 [arXiv:1705.08450] [InSPIRE]. 
[16] M. Garny, J. Heisig, B. Lülf and S. Vogl, Coannihilation without chemical equilibrium, Phys. Rev. D 96 (2017) 103521 [arXiv:1705.09292] [InSPIRE].

[17] M. Garny, J. Heisig, M. Hufnagel and B. Lülf, Top-philic dark matter within and beyond the WIMP paradigm, Phys. Rev. D 97 (2018) 075002 [arXiv:1802.00814] [InSPIRE].

[18] E.D. Carlson, M.E. Machacek and L.J. Hall, Self-interacting dark matter, Astrophys. J. 398 (1992) 43 [INSPIRE].

[19] C. Boehm and P. Fayet, Scalar dark matter candidates, Nucl. Phys. B 683 (2004) 219 [hep-ph/0305261] [INSPIRE].

[20] D.P. Finkbeiner and N. Weiner, Exciting dark matter and the INTEGRAL/SPI $511 \mathrm{keV}$ signal, Phys. Rev. D 76 (2007) 083519 [astro-ph/0702587] [INSPIRE].

[21] M. Pospelov, A. Ritz and M.B. Voloshin, Secluded WIMP dark matter, Phys. Lett. B 662 (2008) 53 [arXiv:0711.4866] [INSPIRE].

[22] J.L. Feng and J. Kumar, The WIMPless miracle: dark-matter particles without weak-scale masses or weak interactions, Phys. Rev. Lett. 101 (2008) 231301 [arXiv:0803.4196] [INSPIRE].

[23] D.E. Kaplan, M.A. Luty and K.M. Zurek, Asymmetric dark matter, Phys. Rev. D 79 (2009) 115016 [arXiv:0901.4117] [INSPIRE].

[24] A. Falkowski, J.T. Ruderman and T. Volansky, Asymmetric dark matter from leptogenesis, JHEP 05 (2011) 106 [arXiv:1101.4936] [INSPIRE].

[25] Y. Hochberg, E. Kuflik, T. Volansky and J.G. Wacker, Mechanism for thermal relic dark matter of strongly interacting massive particles, Phys. Rev. Lett. 113 (2014) 171301 [arXiv: 1402.5143] [INSPIRE].

[26] N. Bernal, C. Garcia-Cely and R. Rosenfeld, WIMP and SIMP dark matter from the spontaneous breaking of a global group, JCAP 04 (2015) 012 [arXiv:1501.01973] [INSPIRE].

[27] R.T. D'Agnolo and A. Hook, Selfish dark matter, Phys. Rev. D 91 (2015) 115020 [arXiv: 1504.00361] [INSPIRE].

[28] E. Kuflik et al., Elastically decoupling dark matter, Phys. Rev. Lett. 116 (2016) 221302 [arXiv: 1512.04545] [INSPIRE].

[29] J.A. Dror, E. Kuflik and W.H. Ng, Codecaying dark matter, Phys. Rev. Lett. 117 (2016) 211801 [arXiv: 1607.03110] [INSPIRE].

[30] J. Kopp et al., Impeded dark matter, JHEP 12 (2016) 033 [arXiv:1609.02147] [INSPIRE].

[31] S. Knapen, T. Lin and K.M. Zurek, Light dark matter: models and constraints, Phys. Rev. D 96 (2017) 115021 [arXiv: 1709.07882] [INSPIRE].

[32] A. Falkowski, E. Kuflik, N. Levi and T. Volansky, Light dark matter from leptogenesis, arXiv: 1712.07652 [INSPIRE].

[33] S. Mizuta and M. Yamaguchi, Coannihilation effects and relic abundance of Higgsino dominant LSP(s), Phys. Lett. B 298 (1993) 120 [hep-ph/9208251] [INSPIRE].

[34] J. Edsjo and P. Gondolo, Neutralino relic density including coannihilations, Phys. Rev. D 56 (1997) 1879 [hep-ph/9704361] [INSPIRE].

[35] J.R. Ellis, T. Falk and K.A. Olive, Neutralino-Stau coannihilation and the cosmological upper limit on the mass of the lightest supersymmetric particle, Phys. Lett. B 444 (1998) 367 [hep-ph/9810360] [INSPIRE]. 
[36] J.R. Ellis, T. Falk, K.A. Olive and M. Srednicki, Calculations of neutralino-stau coannihilation channels and the cosmologically relevant region of MSSM parameter space, Astropart. Phys. 13 (2000) 181 [Erratum ibid. 15 (2001) 413] [hep-ph/9905481] [INSPIRE].

[37] M.E. Gomez, G. Lazarides and C. Pallis, Supersymmetric cold dark matter with Yukawa unification, Phys. Rev. D 61 (2000) 123512 [hep-ph/9907261] [INSPIRE].

[38] C. Boehm, A. Djouadi and M. Drees, Light scalar top quarks and supersymmetric dark matter, Phys. Rev. D 62 (2000) 035012 [hep-ph/9911496] [INSPIRE].

[39] M.E. Gomez, G. Lazarides and C. Pallis, Yukawa unification, $b \rightarrow s \gamma$ and Bino-Stau coannihilation, Phys. Lett. B 487 (2000) 313 [hep-ph/0004028] [inSPIRE].

[40] A. Birkedal-Hansen and B.D. Nelson, The role of Wino content in neutralino dark matter, Phys. Rev. D 64 (2001) 015008 [hep-ph/0102075] [INSPIRE].

[41] R.L. Arnowitt, B. Dutta and Y. Santoso, Coannihilation effects in supergravity and D-brane models, Nucl. Phys. B 606 (2001) 59 [hep-ph/0102181] [InSPIRE].

[42] J.R. Ellis, K.A. Olive and Y. Santoso, Calculations of neutralino stop coannihilation in the CMSSM, Astropart. Phys. 18 (2003) 395 [hep-ph/0112113] [INSPIRE].

[43] H. Baer, C. Balázs and A. Belyaev, Neutralino relic density in minimal supergravity with coannihilations, JHEP 03 (2002) 042 [hep-ph/0202076] [INSPIRE].

[44] T. Nihei, L. Roszkowski and R. Ruiz de Austri, Exact cross-sections for the neutralino slepton coannihilation, JHEP 07 (2002) 024 [hep-ph/0206266] [INSPIRE].

[45] A. Birkedal-Hansen and B.D. Nelson, Relic neutralino densities and detection rates with nonuniversal gaugino masses, Phys. Rev. D 67 (2003) 095006 [hep-ph/0211071] [INSPIRE].

[46] S. Profumo and C.E. Yaguna, Gluino coannihilations and heavy bino dark matter, Phys. Rev. D 69 (2004) 115009 [hep-ph/0402208] [INSPIRE].

[47] H. Baer et al., Mixed wino dark matter: Consequences for direct, indirect and collider detection, JHEP 07 (2005) 046 [hep-ph/0505227] [INSPIRE].

[48] H. Baer et al., Exploring the BWCA (bino-wino co-annihilation) scenario for neutralino dark matter, JHEP 12 (2005) 011 [hep-ph/0511034] [INSPIRE].

[49] N. Arkani-Hamed, A. Delgado and G.F. Giudice, The Well-tempered neutralino, Nucl. Phys. B 741 (2006) 108 [hep-ph/0601041] [InSPIRE].

[50] C. Cheung, L.J. Hall, D. Pinner and J.T. Ruderman, Prospects and blind spots for neutralino dark matter, JHEP 05 (2013) 100 [arXiv:1211.4873] [INSPIRE].

[51] N. Nagata, H. Otono and S. Shirai, Probing bino-gluino coannihilation at the LHC, Phys. Lett. B 748 (2015) 24 [arXiv: 1504.00504] [INSPIRE].

[52] N. Nagata, H. Otono and S. Shirai, Probing Bino-Wino coannihilation at the LHC, JHEP 10 (2015) 086 [arXiv: 1506.08206] [INSPIRE].

[53] S. Tulin, H.-B. Yu and K.M. Zurek, Three exceptions for thermal dark matter with enhanced annihilation to $\gamma \gamma$, Phys. Rev. D 87 (2013) 036011 [arXiv:1208.0009] [INSPIRE].

[54] A. Ibarra, A. Pierce, N.R. Shah and S. Vogl, Anatomy of coannihilation with a scalar top partner, Phys. Rev. D 91 (2015) 095018 [arXiv:1501.03164] [INSPIRE].

[55] M.J. Baker et al., The coannihilation codex, JHEP 12 (2015) 120 [arXiv:1510.03434] [INSPIRE].

[56] V.V. Khoze, A.D. Plascencia and K. Sakurai, Simplified models of dark matter with a long-lived co-annihilation partner, JHEP 06 (2017) 041 [arXiv: 1702.00750] [INSPIRE]. 
[57] S. El Hedri, A. Kaminska, M. de Vries and J. Zurita, Simplified phenomenology for colored dark sectors, JHEP 04 (2017) 118 [arXiv:1703.00452] [INSPIRE].

[58] A. Davoli, A. De Simone, T. Jacques and V. Sanz, Displaced vertices from pseudo-Dirac dark matter, JHEP 11 (2017) 025 [arXiv: 1706.08985] [INSPIRE].

[59] A. Davoli, A. De Simone, T. Jacques and A. Morandini, LHC phenomenology of dark matter with a color-octet partner, JHEP 07 (2018) 054 [arXiv:1803.02861] [INSPIRE].

[60] J.T. Ruderman, Light dark matter from Boltzmann factors, talk given at Beyond WIMPs: From Theory To Detection, May 28-June 1, Ha Goshrim, Israel (2015).

[61] E. Izaguirre, Y. Kahn, G. Krnjaic and M. Moschella, Testing light dark matter coannihilation with fixed-target experiments, Phys. Rev. D 96 (2017) 055007 [arXiv: 1703.06881] [INSPIRE].

[62] B. Chauhan, Sub-MeV self interacting dark matter, Phys. Rev. D 97 (2018) 123017 [arXiv: 1711.02970] [INSPIRE].

[63] M. Battaglieri et al., US Cosmic Visions: New Ideas in Dark Matter 2017: Community Report, arXiv:1707.04591 [INSPIRE].

[64] Planck collaboration, P.A.R. Ade et al., Planck 2015 results. XIII. Cosmological parameters, Astron. Astrophys. 594 (2016) A13 [arXiv:1502.01589] [INSPIRE].

[65] E. Izaguirre, G. Krnjaic, P. Schuster and N. Toro, Physics motivation for a pilot dark matter search at Jefferson Laboratory, Phys. Rev. D 90 (2014) 014052 [arXiv:1403.6826] [INSPIRE].

[66] D. Barducci et al., Collider limits on new physics within MicrOMEGAs_4.3, Comput. Phys. Commun. 222 (2018) 327 [arXiv:1606.03834] [INSPIRE].

[67] J.L. Feng, H. Tu and H.-B. Yu, Thermal relics in hidden sectors, JCAP 10 (2008) 043 [arXiv:0808.2318] [INSPIRE].

[68] A.A. de Laix, R.J. Scherrer and R.K. Schaefer, Constraints of selfinteracting dark matter, Astrophys. J. 452 (1995) 495 [astro-ph/9502087] [INSPIRE].

[69] N. Yamanaka, S. Fujibayashi, S. Gongyo and H. Iida, Dark matter in the hidden gauge theory, arXiv: 1411.2172 [INSPIRE].

[70] N. Bernal et al., Production regimes for self-interacting dark matter, JCAP 03 (2016) 018 [arXiv: 1510.08063] [INSPIRE].

[71] N. Bernal and X. Chu, $\mathbb{Z}_{2}$ SIMP dark matter, JCAP 01 (2016) 006 [arXiv:1510.08527] [INSPIRE].

[72] A. Soni and Y. Zhang, Hidden SU(N) glueball dark matter, Phys. Rev. D 93 (2016) 115025 [arXiv: 1602.00714] [INSPIRE].

[73] D. Pappadopulo, J.T. Ruderman and G. Trevisan, Dark matter freeze-out in a nonrelativistic sector, Phys. Rev. D 94 (2016) 035005 [arXiv: 1602.04219] [INSPIRE].

[74] M. Farina, D. Pappadopulo, J.T. Ruderman and G. Trevisan, Phases of cannibal dark matter, JHEP 12 (2016) 039 [arXiv:1607.03108] [INSPIRE].

[75] J.D. Clarke, R. Foot and R.R. Volkas, Phenomenology of a very light scalar (100 MeV $<m_{h}<10 \mathrm{GeV}$ ) mixing with the SM Higgs, JHEP 02 (2014) 123 [arXiv:1310.8042] [INSPIRE].

[76] J.L. Feng et al., Dark Higgs bosons at the ForwArd Search ExpeRiment, Phys. Rev. D 97 (2018) 055034 [arXiv:1710.09387] [inSPIRE]. 
[77] K. Jedamzik, Big bang nucleosynthesis constraints on hadronically and electromagnetically decaying relic neutral particles, Phys. Rev. D 74 (2006) 103509 [hep-ph/0604251] [INSPIRE].

[78] V. Poulin and P.D. Serpico, Nonuniversal BBN bounds on electromagnetically decaying particles, Phys. Rev. D 91 (2015) 103007 [arXiv:1503.04852] [INSPIRE].

[79] KTEV collaboration, A. Alavi-Harati et al., Search for the Decay $K_{L} \rightarrow \pi^{0} \mu^{+} \mu^{-}$, Phys. Rev. Lett. 84 (2000) 5279 [hep-ex/0001006] [INSPIRE].

[80] KTEV collaboration, A. Alavi-Harati et al., Search for the rare decay $K(L) \rightarrow \pi^{0} e^{+} e^{-}$, Phys. Rev. Lett. 93 (2004) 021805 [hep-ex/0309072] [INSPIRE].

[81] E787 collaboration, S. Adler et al., Further search for the decay $K^{+} \rightarrow \pi^{+} \nu \bar{\nu}$ in the momentum region $P<195 \mathrm{MeV} / c$, Phys. Rev. D 70 (2004) 037102 [hep-ex/0403034] [INSPIRE].

[82] Belle collaboration, K.F. Chen et al., Search for $B \rightarrow h^{(*)} \nu \bar{\nu}$ decays at Belle, Phys. Rev. Lett. 99 (2007) 221802 [arXiv:0707.0138] [INSPIRE].

[83] BNL-E949 collaboration, A.V. Artamonov et al., Study of the decay $K^{+} \rightarrow \pi^{+} \nu \bar{\nu}$ in the momentum region $140<P_{\pi}<199 \mathrm{MeV} / c$, Phys. Rev. D 79 (2009) 092004 [arXiv: 0903.0030] [INSPIRE].

[84] BABAR collaboration, P. del Amo Sanchez et al., Search for the rare decay $B \rightarrow K \nu \bar{\nu}$, Phys. Rev. D 82 (2010) 112002 [arXiv:1009.1529] [INSPIRE].

[85] LHCb collaboration, Search for hidden-sector bosons in $B^{0} \rightarrow K^{* 0} \mu^{+} \mu^{-}$decays, Phys. Rev. Lett. 115 (2015) 161802 [arXiv:1508. 04094] [INSPIRE].

[86] LHCb collaboration, Search for long-lived scalar particles in $B^{+} \rightarrow K^{+} \chi\left(\mu^{+} \mu^{-}\right)$decays, Phys. Rev. D 95 (2017) 071101 [arXiv:1612.07818] [INSPIRE].

[87] CHARM collaboration, F. Bergsma et al., Search for axion like particle production in $400 \mathrm{GeV}$ proton-copper interactions, Phys. Lett. B 157 (1985) 458.

[88] L3 collaboration, M. Acciarri et al., Search for neutral Higgs boson production through the process $e^{+} e^{-} \rightarrow ; Z * H^{0}$, Phys. Lett. B 385 (1996) 454 [inSPIRE].

[89] Delphi, OPAl, AlePh, LeP Working Group for Higgs Boson Searches, L3 collaboration, S. Schael et al., Search for neutral MSSM Higgs bosons at LEP, Eur. Phys. J. C 47 (2006) 547 [hep-ex/0602042] [INSPIRE].

[90] XENON1T collaboration, E. Aprile, The XENON1T dark matter search experiment, Springer Proc. Phys. 148 (2013) 93 [arXiv:1206.6288] [INSPIRE].

[91] XENON collaboration, E. Aprile et al., Physics reach of the XENON1T dark matter experiment, JCAP 04 (2016) 027 [arXiv: 1512.07501] [INSPIRE].

[92] DARWIN collaboration, J. Aalbers et al., DARWIN: towards the ultimate dark matter detector, JCAP 11 (2016) 017 [arXiv:1606.07001] [INSPIRE].

[93] B.J. Mount et al., LUX-ZEPLIN (LZ) technical design report, arXiv:1703.09144 [INSPIRE].

[94] V.V. Gligorov et al., Searching for long-lived particles: a compact detector for exotics at LHCb, Phys. Rev. D 97 (2018) 015023 [arXiv:1708.09395] [INSPIRE].

[95] J.A. Evans, S. Gori and J. Shelton, Looking for the WIMP next door, JHEP 02 (2018) 100 [arXiv: 1712.03974] [INSPIRE]. 
[96] J.L. Feng, I. Galon, F. Kling and S. Trojanowski, ForwArd Search ExpeRiment at the LHC, Phys. Rev. D 97 (2018) 035001 [arXiv:1708.09389] [inSPIRE].

[97] J.P. Chou, D. Curtin and H.J. Lubatti, New Detectors to Explore the Lifetime Frontier, Phys. Lett. B 767 (2017) 29 [arXiv:1606.06298] [INSPIRE].

[98] J.A. Evans, Detecting hidden particles with MATHUSLA, Phys. Rev. D 97 (2018) 055046 [arXiv: 1708.08503] [INSPIRE].

[99] S. Alekhin et al., A facility to Search for Hidden particles at the CERN SPS: the SHiP physics case, Rept. Prog. Phys. 79 (2016) 124201 [arXiv: 1504.04855] [INSPIRE].

[100] SHIP collaboration, M. Anelli et al., A facility to Search for Hidden Particles (SHiP) at the CERN SPS, arXiv:1504.04956 [INSPIRE].

[101] CMB-S4 collaboration, K.N. Abazajian et al., CMB-S4 science book, first edition, arXiv: 1610.02743 [INSPIRE].

[102] C. Boehm, M.J. Dolan and C. McCabe, A lower bound on the mass of cold thermal dark matter from Planck, JCAP 08 (2013) 041 [arXiv: 1303.6270] [INSPIRE].

[103] A. Berlin and N. Blinov, Thermal dark matter below an MeV, Phys. Rev. Lett. 120 (2018) 021801 [arXiv:1706.07046] [INSPIRE].

[104] X.-L. Chen and M. Kamionkowski, Particle decays during the cosmic dark ages, Phys. Rev. D 70 (2004) 043502 [astro-ph/0310473] [INSPIRE].

[105] N. Padmanabhan and D.P. Finkbeiner, Detecting dark matter annihilation with CMB polarization: signatures and experimental prospects, Phys. Rev. D 72 (2005) 023508 [astro-ph/0503486] [INSPIRE].

[106] T.R. Slatyer, Indirect dark matter signatures in the cosmic dark ages. I. Generalizing the bound on s-wave dark matter annihilation from Planck results, Phys. Rev. D 93 (2016) 023527 [arXiv: 1506.03811] [INSPIRE].

[107] T.R. Slatyer, Indirect dark matter signatures in the cosmic dark ages II. Ionization, heating and photon production from arbitrary energy injections, Phys. Rev. D 93 (2016) 023521 [arXiv: 1506.03812] [INSPIRE].

[108] B. Batell, T. Han, D. McKeen and B. Shams Es Haghi, Thermal Dark Matter Through the Dirac Neutrino Portal, Phys. Rev. D 97 (2018) 075016 [arXiv: 1709.07001] [inSPIRE].

[109] M. Schmaltz and N. Weiner, A portalino to the dark sector, arXiv:1709.09164 [INSPIRE].

[110] G. Elor, N.L. Rodd, T.R. Slatyer and W. Xue, Model-independent indirect detection constraints on hidden sector dark matter, JCAP 06 (2016) 024 [arXiv:1511.08787] [INSPIRE].

[111] A. Fradette and M. Pospelov, BBN for the LHC: constraints on lifetimes of the Higgs portal scalars, Phys. Rev. D 96 (2017) 075033 [arXiv:1706.01920] [INSPIRE].

[112] B. Henning and H. Murayama, Constraints on light dark matter from Big Bang nucleosynthesis, arXiv:1205.6479 [INSPIRE].

[113] M. Kawasaki, K. Kohri, T. Moroi and Y. Takaesu, Revisiting Big-Bang nucleosynthesis constraints on long-lived decaying particles, Phys. Rev. D 97 (2018) 023502 [arXiv: 1709.01211] [INSPIRE].

[114] BELLE collaboration, J.T. Wei et al., Measurement of the differential branching fraction and forward-backword asymmetry for $B \rightarrow K^{(*)} \ell^{+} \ell^{-}$, Phys. Rev. Lett. 103 (2009) 171801 [arXiv: 0904.0770] [INSPIRE]. 
[115] K. Schmidt-Hoberg, F. Staub and M.W. Winkler, Constraints on light mediators: confronting dark matter searches with B physics, Phys. Lett. B 727 (2013) 506 [arXiv:1310.6752] [INSPIRE].

[116] T. Flacke et al., Phenomenology of relaxion-Higgs mixing, JHEP 06 (2017) 050 [arXiv: 1610.02025] [INSPIRE].

[117] F. Bezrukov and D. Gorbunov, Light inflaton Hunter's guide, JHEP 05 (2010) 010 [arXiv: 0912.0390] [INSPIRE].

[118] E. Izaguirre, G. Krnjaic, P. Schuster and N. Toro, New electron beam-dump experiments to search for MeV to few-GeV dark matter, Phys. Rev. D 88 (2013) 114015 [arXiv: 1307.6554] [INSPIRE].

[119] BDX collaboration, M. Battaglieri et al., Dark Matter Search in a Beam-Dump eXperiment (BDX) at Jefferson Lab, arXiv:1607.01390 [INSPIRE].

[120] NA64 collaboration, D. Banerjee et al., Search for invisible decays of sub-GeV dark photons in missing-energy events at the CERN SPS, Phys. Rev. Lett. 118 (2017) 011802 [arXiv: 1610.02988] [INSPIRE].

[121] MiniBooNE collaboration, A.A. Aguilar-Arevalo et al., Dark matter search in a proton beam dump with MiniBooNE, Phys. Rev. Lett. 118 (2017) 221803 [arXiv:1702.02688] [INSPIRE].

[122] G. Krnjaic, Probing light thermal dark-matter with a Higgs portal mediator, Phys. Rev. D 94 (2016) 073009 [arXiv: 1512.04119] [INSPIRE].

[123] J.R. Bond, G. Efstathiou and J. Silk, Massive neutrinos and the large scale structure of the universe, Phys. Rev. Lett. 45 (1980) 1980 [InSPIRE].

[124] WMAP collaboration, D.N. Spergel et al., First year Wilkinson Microwave Anisotropy Probe (WMAP) observations: determination of cosmological parameters, Astrophys. J. Suppl. 148 (2003) 175 [astro-ph/0302209] [INSPIRE].

[125] SDSS collaboration, M. Tegmark et al., Cosmological parameters from SDSS and WMAP, Phys. Rev. D 69 (2004) 103501 [astro-ph/0310723] [INSPIRE].

[126] S. Hannestad and G. Raffelt, Cosmological mass limits on neutrinos, axions and other light particles, JCAP 04 (2004) 008 [hep-ph/0312154] [INSPIRE].

[127] P. Crotty, J. Lesgourgues and S. Pastor, Current cosmological bounds on neutrino masses and relativistic relics, Phys. Rev. D 69 (2004) 123007 [hep-ph/0402049] [InSPIRE].

[128] SDSS collaboration, U. Seljak et al., Cosmological parameter analysis including SDSS Ly-alpha forest and galaxy bias: constraints on the primordial spectrum of fluctuations, neutrino mass and dark energy, Phys. Rev. D 71 (2005) 103515 [astro-ph/0407372] [INSPIRE].

[129] M. Viel et al., Constraining warm dark matter candidates including sterile neutrinos and light gravitinos with WMAP and the Lyman- $\alpha$ forest, Phys. Rev. D 71 (2005) 063534 [astro-ph/0501562] [INSPIRE].

[130] J. Lesgourgues and S. Pastor, Massive neutrinos and cosmology, Phys. Rept. 429 (2006) 307 [astro-ph/0603494] [inSPIRE].

[131] D. Hooper et al., MeV dark matter and small scale structure, Phys. Rev. D 76 (2007) 103515 [arXiv: 0704.2558] [INSPIRE].

[132] D.N. Spergel and P.J. Steinhardt, Observational evidence for selfinteracting cold dark matter, Phys. Rev. Lett. 84 (2000) 3760 [astro-ph/9909386] [INSPIRE]. 
[133] M. Markevitch et al., Direct constraints on the dark matter self-interaction cross-section from the merging galaxy cluster 1E0657-56, Astrophys. J. 606 (2004) 819 [astro-ph/0309303] [INSPIRE].

[134] D. Clowe, A. Gonzalez and M. Markevitch, Weak lensing mass reconstruction of the interacting cluster 1E0657-558: direct evidence for the existence of dark matter, Astrophys. J. 604 (2004) 596 [astro-ph/0312273] [INSPIRE].

[135] S.W. Randall et al., Constraints on the self-interaction cross-section of dark matter from numerical simulations of the merging galaxy cluster 1E 0657-56, Astrophys. J. 679 (2008) 1173 [arXiv: 0704.0261] [INSPIRE].

[136] M. Rocha et al., Cosmological simulations with self-interacting dark matter I: constant density cores and substructure, Mon. Not. Roy. Astron. Soc. 430 (2013) 81 [arXiv:1208.3025] [INSPIRE].

[137] A.H.G. Peter, M. Rocha, J.S. Bullock and M. Kaplinghat, Cosmological simulations with self-interacting dark matter II: halo shapes vs. observations, Mon. Not. Roy. Astron. Soc. 430 (2013) 105 [arXiv:1208.3026] [INSPIRE].

[138] D. Harvey et al., The non-gravitational interactions of dark matter in colliding galaxy clusters, Science 347 (2015) 1462 [arXiv: 1503. 07675] [INSPIRE].

[139] M. Viel et al., Warm dark matter as a solution to the small scale crisis: new constraints from high redshift Lyman- $\alpha$ forest data, Phys. Rev. D 88 (2013) 043502 [arXiv:1306.2314] [INSPIRE].

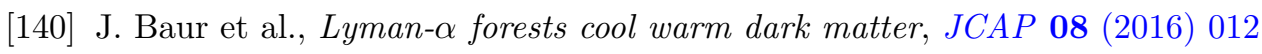
[arXiv: 1512.01981] [INSPIRE].

[141] V. Iršič et al., New Constraints on the free-streaming of warm dark matter from intermediate and small scale Lyman- $\alpha$ forest data, Phys. Rev. D 96 (2017) 023522 [arXiv: 1702.01764] [INSPIRE].

[142] C. Yèche et al., Constraints on neutrino masses from Lyman-alpha forest power spectrum with BOSS and XQ-100, JCAP 06 (2017) 047 [arXiv: 1702.03314] [INSPIRE].

[143] A. Loeb and M. Zaldarriaga, The Small-scale power spectrum of cold dark matter, Phys. Rev. D 71 (2005) 103520 [astro-ph/0504112] [INSPIRE].

[144] T. Binder et al., Matter power spectrum in hidden neutrino interacting dark matter models: a closer look at the collision term, JCAP 11 (2016) 043 [arXiv: 1602.07624] [INSPIRE].

[145] T. Bringmann, H.T. Ihle, J. Kersten and P. Walia, Suppressing structure formation at dwarf galaxy scales and below: late kinetic decoupling as a compelling alternative to warm dark matter, Phys. Rev. D 94 (2016) 103529 [arXiv: 1603.04884] [INSPIRE].

[146] P. Adshead, Y. Cui and J. Shelton, Chilly dark sectors and asymmetric reheating, JHEP 06 (2016) 016 [arXiv: 1604.02458] [INSPIRE].

[147] S. Tremaine and J.E. Gunn, Dynamical role of light neutral leptons in cosmology, Phys. Rev. Lett. 42 (1979) 407 [INSPIRE].

[148] A. Boyarsky, O. Ruchayskiy and D. Iakubovskyi, A Lower bound on the mass of Dark Matter particles, JCAP 03 (2009) 005 [arXiv: 0808.3902] [INSPIRE].

[149] L. Darmé, S. Rao and L. Roszkowski, Light dark Higgs boson in minimal sub-GeV dark matter scenarios, JHEP 03 (2018) 084 [arXiv: 1710.08430] [INSPIRE].

[150] R. Essig et al., Constraining light dark matter with low-energy $e^{+} e^{-}$colliders, JHEP 11 (2013) 167 [arXiv: 1309.5084] [INSPIRE]. 
[151] BABAR collaboration, J.P. Lees et al., Search for invisible decays of a dark photon produced in $e^{+} e^{-}$collisions at BaBar, Phys. Rev. Lett. 119 (2017) 131804 [arXiv:1702.03327] [INSPIRE].

[152] J.D. Bjorken et al., Search for neutral metastable penetrating particles produced in the SLAC beam dump, Phys. Rev. D 38 (1988) 3375 [InSPIRE].

[153] LSND collaboration, L.B. Auerbach et al., Measurement of electron-neutrino-electron elastic scattering, Phys. Rev. D 63 (2001) 112001 [hep-ex/0101039] [INSPIRE].

[154] V. Poulin, J. Lesgourgues and P.D. Serpico, Cosmological constraints on exotic injection of electromagnetic energy, JCAP 03 (2017) 043 [arXiv: 1610.10051] [INSPIRE].

[155] A. Kogut et al., The Primordial Inflation Explorer (PIXIE): a nulling polarimeter for cosmic microwave background observations, JCAP 07 (2011) 025 [arXiv: 1105.2044] [INSPIRE].

[156] J. Chluba and D. Jeong, Teasing bits of information out of the CMB energy spectrum, Mon. Not. Roy. Astron. Soc. 438 (2014) 2065 [arXiv:1306.5751] [INSPIRE].

[157] M.H. Abitbol et al., Prospects for measuring cosmic microwave background spectral distortions in the presence of foregrounds, Mon. Not. Roy. Astron. Soc. 471 (2017) 1126 [arXiv: 1705.01534] [INSPIRE].

[158] E. Izaguirre et al., Testing GeV-scale dark matter with fixed-target missing momentum experiments, Phys. Rev. D 91 (2015) 094026 [arXiv:1411.1404] [INSPIRE].

[159] Particle Data Group collaboration, J. Beringer et al., Review of particle physics (RPP), Phys. Rev. D 86 (2012) 010001 [INSPIRE].

[160] BDX collaboration, M. Battaglieri et al., Dark matter search in a Beam-Dump eXperiment (BDX) at Jefferson Lab, arXiv:1406.3028 [INSPIRE].

[161] MiniBooNE collaboration, A. Bazarko, MiniBooNE: Status of the booster neutrino experiment, Nucl. Phys. Proc. Suppl. 91 (2001) 210 [hep-ex/0009056] [INSPIRE].

[162] MiniBooNE collaboration, A.A. Aguilar-Arevalo et al., The MiniBooNE Detector, Nucl. Instrum. Meth. A 599 (2009) 28 [arXiv:0806.4201] [INSPIRE]. 